\title{
Water-Soluble Constituents of Caraway: Carvone Derivatives and Their Glucosides
}

\author{
Tetsuko Matsumura, Toru Ishikawa, and Junichi Kitajima* \\ Showa Pharmaceutical University, Higashi-Tamagawagakuen 3, Machida, Tokyo 194-8543, Japan. \\ Received August 10, 2001; accepted October 11, 2001
}

\begin{abstract}
Nine monoterpenoids related to carvone and seven glucosides were isolated from the water-soluble portion of the methanolic extract of the caraway (fruit of Carum carvi L.), and their structures were clarified by spectral investigation. Among them, eight monoterpenoids and six glucosides were new.
\end{abstract}

Key words caraway; Carum carvi fruit; carvone derivative; $p$-menthanediol; $p$-menthanetetrol; $p$-menthane glucoside

In a previous paper, we reported the isolation and characterization of 10 new $p$-menthanetriols, including eight stereoisomers of $p$-menthane-2,8,9-triol and five new glucosides from the methanolic extract of caraway (fruit of Carum carvi LinN., Umbelliferae), which has been used as a popular aromatic herb and medicine. ${ }^{1,2)}$ In continuation of our studies on the water-soluble constituents of spices, and to reveal the relationship between the essential oil and the water-soluble constituents, ${ }^{1,3)}$ we undertook the isolation and structure elucidation of monoterpenoids related to carvone, and their glucosides.

Commercial caraway was extracted with $70 \%$ methanol, and the methanolic extract was worked up as described in the previous paper. ${ }^{1)}$ From the same aqueous portion, monoterpenoid diol (1), monoterpenoid enone-diols $(\mathbf{2}, \mathbf{3})$, monoterpenoid tetrols (4 to 9), and monoterpenoid glucosides (10 to 16) were isolated by the combination of Sephadex LH-20, silica gel, Lobar RP-8 column chromatography, and HPLC. Among them, eight monoterpenoids ( 2 to 9 ) and six monoterpenoid glucosides (10 to $\mathbf{1 5})$ were new. All new glucosides described in this paper were $\beta$-D-glucopyranosides as shown by their ${ }^{13} \mathrm{C}-\mathrm{NMR}$ data (Table 1 ), and this was confirmed by hydrolysis to yield D-glucose, or by comparison of the $[\alpha]_{\mathrm{D}}$ or $[M]_{\mathrm{D}}$ values with those of their aglycones. ${ }^{4)}$ Their molecular formulae were suggested from the accurate mass number of $[\mathrm{M}+\mathrm{H}]^{+}$or $[\mathrm{M}+\mathrm{Na}]^{+}$ion peaks in the high-resolution positive $\mathrm{FAB}-\mathrm{MS}$.

Diol $1\left(\mathrm{C}_{10} \mathrm{H}_{18} \mathrm{O}_{2}\right.$, an amorphous powder, $\left.[\alpha]_{\mathrm{D}}^{21}+34^{\circ}\right)$ and glucoside $16\left(\mathrm{C}_{16} \mathrm{H}_{28} \mathrm{O}_{7}\right.$, an amorphous powder, $\left.[\alpha]_{\mathrm{D}}^{25}-50^{\circ}\right)$ were identified as $(1 S, 2 S, 4 R)$ - $p$-menth-8-ene-1,2-diol ${ }^{5)}$ and $(4 S)$ - $p$-menth-1-ene-7,8-diol 8-O- $\beta$-D-glucopyranoside, ${ }^{6)}$ respectively.

Glucoside $10\left(\mathrm{C}_{16} \mathrm{H}_{28} \mathrm{O}_{7}\right.$, mp $\left.154-156^{\circ} \mathrm{C},[\alpha]_{\mathrm{D}}^{25}+13^{\circ}\right)$ showed $[\mathrm{M}+\mathrm{H}]^{+}$ion peak at $\mathrm{m} / z \quad 333$ and [M$\left.\mathrm{C}_{6} \mathrm{H}_{12} \mathrm{O}_{6}+\mathrm{H}\right]^{+}$ion peak at $m / z 153$ in the positive FAB-MS. Glucoside $\mathbf{1 0}$ was hydrolyzed with hesperidinase and, from the hydrolyzed mixtures, 1 and D-glucose were obtained. Consequently, $\mathbf{1 0}$ was a monoglucoside of $\mathbf{1}$. The position of the $\beta$-glucosyl unit of $\mathbf{1 0}$ was proved to be $\mathrm{C}-2$ from the cross-peak between the glucosyl $\mathrm{H}-1 / \mathrm{C}-2$ in the heteronuclear multiple bond connectivity (HMBC) spectrum and the observed nuclear Overhauser effect (NOE) interaction between the glucosyl $\mathrm{H}-1 / \mathrm{H}-2$ in the NOE spectroscopy (NOESY) spectrum. Thus $\mathbf{1 0}$ was characterized as $(1 S, 2 S, 4 R)$ $p$-menth-8-ene-1,2-diol 2- $O$ - $\beta$-D-glucopyranoside.

Enone-diol $2\left(\mathrm{C}_{10} \mathrm{H}_{16} \mathrm{O}_{3}\right.$, an amorphous powder, $[\alpha]_{\mathrm{D}}^{25}$ $\left.-6^{\circ}\right)$ and $3\left(\mathrm{C}_{10} \mathrm{H}_{16} \mathrm{O}_{3}\right.$, an amorphous powder, $\left.[\alpha]_{\mathrm{D}}^{25}-7^{\circ}\right)$ showed an $[\mathrm{M}+\mathrm{H}]^{+}$ion peak at $m / z 185$ in the positive FABMS. They showed similar ${ }^{1} \mathrm{H}$ - and ${ }^{13} \mathrm{C}-\mathrm{NMR}$ spectra (Tables $2,1)$, and have two tert-methyls, one hydroxymethyl, two methylenes, one methine, one oxygenated quaternary carbon, and one carbonyl group conjugated with a trisubstituted double bond. From analysis of the HMBC correlation data of $\mathbf{3}$ (H-6/C-4, C-5, C-7; H 3 -7/C-1, C-2, C-6; H $-9 / \mathrm{C}-4$, C-8, C$\left.10 ; \mathrm{H}_{3}-10 / \mathrm{C}-4, \mathrm{C}-8, \mathrm{C}-9\right)$, they were suggested to be 8 ,9-dihydroxy-8,9-dihydro derivatives of carvone. Therefore $\mathbf{2}$ and 3 were revealed to be stereoisomers of $\mathrm{C}-8$. In a previous paper, we reported that comparison of $\mathrm{C}-3$ and $\mathrm{C}-5{ }^{13} \mathrm{C}-$ chemical shifts was useful to determine the $\mathrm{C}-8$ configuration of 8-epimeric pairs of $p$-menthane-2,8,9-triol. ${ }^{1)}$ In these pairs, C-3 signals in the $\left(4 R^{*}, 8 S^{*}\right)$-forms were found significantly downfield from those in the $\left(4 R^{*}, 8 R^{*}\right)$-forms; on the contrary, the $\mathrm{C}-5$ signals in $\left(4 R^{*}, 8 S^{*}\right)$-forms appeared significantly upfield from those in the $\left(4 R^{*}, 8 R^{*}\right)$-forms. For 2 and 3, the ${ }^{13} \mathrm{C}$ chemical shift at $\mathrm{C}-3$ of $2(\delta 40.2)$ was downfield from that of $\mathbf{3}(\delta 39.6)$, whereas the C-5 of $\mathbf{2}(\delta 27.2)$ was upfield from that of $\mathbf{3}(\delta 27.8)$. Therfore the stereochemical relationship between C-4 and C-8 was considered to be $4 R^{*}, 8 S^{*}$ in 2 and $4 R^{*}, 8 R^{*}$ in 3 . Comparison of the ${ }^{1} \mathrm{H}-\mathrm{chem}-$ ical shifts of the $\mathrm{H}_{2}-3$ and $\mathrm{H}_{2}-5$ signals of $\mathbf{2}$ and $\mathbf{3}$ was also useful to determine the relative configuration at $\mathrm{C}-8$. As in the case of the 8-epimeric pairs of $p$-menthane-2,8,9-triol, the $\mathrm{H}_{2}-3$ signals of $2\left(\mathrm{H}-3_{\mathrm{ax}} \delta 2.58 ; \mathrm{H}-3_{\text {eq }} \delta 2.98\right)$ were shifted upfield from those of $3\left(\mathrm{H}-3_{\mathrm{ax}} \delta 2.63 ; \mathrm{H}-3_{\mathrm{eq}} \delta 3.12\right)$, and the $\mathrm{H}_{2}-5$ signals of the $\left(4 R^{*}, 8 S^{*}\right)$-form $\left(2 ; \mathrm{H}-5_{\mathrm{ax}} \delta 2.50\right.$; $\mathrm{H}-5_{\mathrm{eq}} \delta$ 2.66) were shifted downfield from those of the $\left(4 R^{*}, 8 R^{*}\right)$-form $\left(3 ; \mathrm{H}-5_{\mathrm{ax}} \delta 2.39 ; \mathrm{H}-5_{\mathrm{eq}} \delta 2.47\right){ }^{1)}$ The absolute configuration of C-4 was determined to be $S$ by the results of circular dichroism (CD) spectra which showed a negative Cotton effect $[250 \mathrm{~nm}$ for $2(\Delta \varepsilon-0.48)$ and $240 \mathrm{~nm}$ for $3(\Delta \varepsilon-0.39)]$, as observed for $(+)$-carvone $[260 \mathrm{~nm}(\Delta \varepsilon$ $-0.41)]^{7)}$ Furthermore, the NOE interactions observed in the one-dimensional (1D)-NOESY spectrum (2; between $\mathrm{H}-$ $3_{\mathrm{ax}} / \mathrm{H}-5_{\mathrm{ax}}$, between $\mathrm{H}_{2}-9 / \mathrm{H}-3_{\text {eq }}, \mathrm{H}-4, \mathrm{H}-5_{\text {eq }}$, and between $\mathrm{H}_{3}$ $10 / \mathrm{H}-3_{\mathrm{ax}}, \mathrm{H}-3_{\mathrm{eq}}, \mathrm{H}-4,3$; between $\mathrm{H}-3_{\mathrm{ax}} / \mathrm{H}-5_{\mathrm{ax}}$, between $\mathrm{H}_{2}-$ 9/H- $3_{\mathrm{ax}}, \mathrm{H}-3_{\mathrm{eq}}$, and between $\mathrm{H}_{3}-10 / \mathrm{H}-3_{\mathrm{ax}}, \mathrm{H}-4, \mathrm{H}-5_{\mathrm{eq}}$; Fig. 1) supported the stereochemical structures. Thus $\mathbf{2}$ and $\mathbf{3}$ were characterized as $(4 S, 8 R)$-8,9-dihydroxy-8,9-dihydrocarvone and $(4 S, 8 S)$-8,9-dihydroxy-8,9-dihydrocarvone, respectively.

Tetrol $4\left(\mathrm{C}_{10} \mathrm{H}_{20} \mathrm{O}_{4}\right.$, an amorphous powder, $\left.[\alpha]_{\mathrm{D}}^{21}+4^{\circ}\right)$ showed an $[\mathrm{M}+\mathrm{H}]^{+}$ion peak at $m / z 205$ in the positive FABMS, and the ${ }^{1} \mathrm{H}$ - and ${ }^{13} \mathrm{C}-\mathrm{NMR}$ spectral data (Tables 2, 1) re- 
Table 1. ${ }^{13} \mathrm{C}-\mathrm{NMR}$ Chemical Shifts of $\mathbf{1}-\mathbf{1 5}, \mathbf{1 4 a}$, and 15a (in Pyridine- $d_{5}, 125 \mathrm{MHz}$ )

\begin{tabular}{|c|c|c|c|c|c|c|c|c|c|}
\hline & 1 & 2 & 3 & 4 & 5 & 6 & 7 & 8 & 9 \\
\hline C-1 & 70.70 & 134.87 & 134.96 & 42.90 & 70.88 & 70.87 & 70.89 & 70.91 & 73.56 \\
\hline $\mathrm{C}-2$ & 74.03 & 199.88 & 200.23 & $71.49^{a)}$ & 74.38 & 74.34 & 75.91 & 76.02 & 77.95 \\
\hline C-3 & 35.39 & 40.17 & 39.58 & 29.37 & 31.41 & 30.47 & 32.97 & 31.91 & 33.87 \\
\hline C-4 & 38.44 & 42.43 & 42.28 & 40.08 & 38.01 & 37.87 & 44.27 & 44.15 & 43.91 \\
\hline C-5 & 27.31 & 27.24 & 27.77 & 30.30 & 22.35 & 23.30 & 22.09 & 23.13 & 24.23 \\
\hline C-6 & 34.69 & 145.93 & 145.44 & $71.51^{a)}$ & 34.92 & 34.88 & 38.63 & 38.64 & 39.74 \\
\hline $\mathrm{C}-7$ & 28.64 & 15.88 & 15.86 & 5.96 & 28.89 & 28.86 & 28.16 & 28.12 & 19.75 \\
\hline C-8 & 151.30 & 73.09 & 73.13 & 74.01 & 74.38 & 74.44 & 74.12 & 74.05 & 73.84 \\
\hline C-9 & 108.69 & 68.55 & 68.58 & 69.12 & 69.11 & 69.21 & 69.08 & 69.15 & 69.07 \\
\hline C-10 & 21.21 & 22.23 & 21.96 & 21.92 & 21.96 & 21.57 & 21.72 & 21.53 & 21.98 \\
\hline
\end{tabular}

\begin{tabular}{|c|c|c|c|c|c|c|c|c|}
\hline & 10 & 11 & 12 & 13 & 14 & $14 a$ & 15 & $15 \mathbf{a}$ \\
\hline C-1 & $70.45(-0.3)$ & $70.27(-0.6)$ & $70.24(-0.6)$ & 73.52 & $30.29(-4.5)$ & 34.75 & $137.35(-3.5)$ & 140.89 \\
\hline $\mathrm{C}-2$ & $83.65(+9.6)$ & $85.43(+11.1)$ & $85.32(+11.0)$ & 77.92 & $78.01(+5.9)$ & 72.09 & 63.91 & 64.48 \\
\hline $\mathrm{C}-3$ & $33.53(-1.9)$ & $29.49(-1.9)$ & $28.24(-2.2)$ & 33.87 & $33.31(-2.3)$ & 35.58 & 37.44 & 37.66 \\
\hline C-4 & 38.64 & 38.15 & 37.24 & 44.00 & 40.47 & 40.59 & 36.01 & 36.19 \\
\hline C-5 & 26.95 & 22.27 & 23.10 & 24.18 & 25.94 & 25.88 & 31.28 & 31.19 \\
\hline C-6 & 34.99 & 35.46 & 35.16 & 39.73 & 30.88 & 31.27 & 127.69 & 124.13 \\
\hline $\mathrm{C}-7$ & 28.07 & 28.74 & 28.66 & 19.81 & 11.38 & 11.37 & $71.69(+6.8)$ & 64.86 \\
\hline C-8 & 150.88 & 74.56 & 74.73 & $73.36(-0.5)$ & 155.06 & 155.49 & 149.93 & 150.17 \\
\hline C-9 & 108.73 & 68.73 & 69.69 & $77.65(+8.6)$ & 107.05 & 106.95 & 109.26 & 109.16 \\
\hline C-10 & 21.25 & 22.06 & 20.77 & 21.91 & 64.32 & 64.50 & 20.92 & 20.92 \\
\hline Glc-1 & 106.25 & 106.46 & 106.11 & 106.09 & 101.46 & & 103.60 & \\
\hline Glc-2 & 75.64 & 75.70 & 75.63 & 75.32 & 75.40 & & 75.22 & \\
\hline Glc-3 & 78.65 & 78.72 & 78.69 & 78.72 & 78.69 & & 78.73 & \\
\hline Glc-4 & 71.74 & 71.83 & 72.06 & 71.69 & 71.74 & & 71.89 & \\
\hline Glc-5 & 78.21 & 78.39 & 78.46 & 78.62 & 78.47 & & 78.52 & \\
\hline Glc-6 & 62.85 & 63.12 & 63.28 & 62.81 & 62.81 & & 62.92 & \\
\hline
\end{tabular}

$\delta$ in ppm from tetramethylsilane (TMS). $\Delta \delta(\delta$ glucoside $-\delta$ aglycone $)$ are given in parentheses. $a$ ) Assignments may be interchanged.

vealed the presence of one tert-methyl, one sec-methyl, one hydroxymethyl, two methylenes, four methines (two oxygenated), and one oxygenated quaternary carbon. From the analysis of HMBC spectral data $\left(\mathrm{H}-4_{\mathrm{ax}} / \mathrm{C}-2, \mathrm{C}-3, \mathrm{C}-5, \mathrm{C}-6\right.$, C-8, C-9, C-10; $\mathrm{H}_{3}-7 / \mathrm{C}-1, \mathrm{C}-2, \mathrm{C}-6 ; \mathrm{H}_{2}-9 / \mathrm{C}-4, \mathrm{C}-8, \mathrm{C}-10$; $\left.\mathrm{H}_{3}-10 / \mathrm{C}-4, \mathrm{C}-8, \mathrm{C}-9\right), 4$ was suggested to be $p$-menthane2,6,8,9-tetrol. As cross-peaks based on the NOE interactions between $\mathrm{H}_{3}-7 / \mathrm{H}-3_{\mathrm{ax}}, \mathrm{H}-5_{\mathrm{ax}}$, between $\mathrm{H}-3_{\mathrm{ax}} / \mathrm{H}-5_{\mathrm{ax}}$, and between $\mathrm{H}-4 / \mathrm{H}-2_{\mathrm{ax}}$, H- $6_{\mathrm{ax}}$ were observed in the NOESY spectrum (Fig. 1), the conformation of the cyclohexane ring was indicated to be the chair form with a 7,8-cis substituent, and the C-2 and C-6 hydroxyl groups were equatorial. The broad H-2 and H-6 signals (each $\delta 4.21$, ddd $J=3.5,3.5,12.0 \mathrm{~Hz}$ ) also suggested the presence of an equatorial hydroxyl group. Therefore 4 was characterized as $4 \beta \mathrm{H}$-cis- $p$-menthane$2 \alpha, 6 \alpha, 8,9$-tetrol.

Tetrols $5\left(\mathrm{C}_{10} \mathrm{H}_{20} \mathrm{O}_{4}\right.$, an amorphous powder, $\left.[\alpha]_{\mathrm{D}}^{22}+30^{\circ}\right)$ and $6\left(\mathrm{C}_{10} \mathrm{H}_{20} \mathrm{O}_{4}\right.$, an amorphous powder, $\left.[\alpha]_{\mathrm{D}}^{22}+27^{\circ}\right)$ revealed an $[\mathrm{M}+\mathrm{H}]^{+}$ion peak at $m / z 205$ in the positive FABMS, and showed similar ${ }^{1} \mathrm{H}-$ and ${ }^{13} \mathrm{C}$-NMR spectral features (Tables 2, 1). They have two tert-methyls, one hydroxymethyl, three methylenes, two methines (one oxygenated), and two oxygenated quaternary carbons. From the result of the HMBC experiment on $5\left(\mathrm{H}-4{ }_{\mathrm{ax}} / \mathrm{C}-5, \mathrm{C}-9\right.$; $\mathrm{H}_{3}-7 / \mathrm{C}-1, \mathrm{C}-2$, C-6; $\left.\mathrm{H}_{2}-9 / \mathrm{C}-4, \mathrm{C}-8, \mathrm{C}-10 ; \mathrm{H}_{3}-10 / \mathrm{C}-4, \mathrm{C}-8, \mathrm{C}-9\right)$, they were suggested to be $p$-menthane-1,2,8,9-tetrol. The conformation of 5 and $\mathbf{6}$ was found to be the 7,8-trans form from the observed NOE interactions between $\mathrm{H}_{3}-7 / \mathrm{H}-6_{\mathrm{ax}}, \mathrm{H}-6_{\text {eq }}$, and between $\mathrm{H}-4 / \mathrm{H}-6_{\mathrm{ax}}$ in their NOESY spectra (Fig. 1), and the configuration of the C-2 hydroxyl was suggested to be axial by the equatorial H-2 signal which found a narrow double doublet with a half-bandwidth of $3 \mathrm{~Hz}$ in their ${ }^{1} \mathrm{H}-\mathrm{NMR}$ spectra. Therefore $\mathbf{5}$ and $\mathbf{6}$ were revealed to be stereoisomers of trans- $p$-menthane- $1,2_{\mathrm{ax}}, 8,9$-tetrol at $\mathrm{C}$-8, respectively. Furthermore, the ${ }^{13} \mathrm{C}$ chemical shift of the $\mathrm{C}-3$ of $5(\delta 31.4)$ was downfield from that of 6 ( $\delta 30.5)$, and the C-5 of 5 ( $\delta 22.3)$ appeared upfield from that of $\mathbf{6}(\delta 23.3)$, with the same relationship as that between $\mathbf{2}$ and $\mathbf{3}$. This conclusion was supported by the results of NOESY spectra of $\mathbf{5}$ and $\mathbf{6}$, which showed the same interactions as the $\left(4 R^{*}, 8 S^{*}\right)$-form of $p$ menthane-2,8,9-triols (between $\mathrm{H}_{3}-10 / \mathrm{H}_{2}-3, \mathrm{H}_{2}-5$, and between $\left.\mathrm{H}_{2}-9 / \mathrm{H}-3_{\mathrm{eq}}\right)$ and the $\left(4 R^{*}, 8 R^{*}\right)$-form of $p$-menthane2,8,9-triols (between $\mathrm{H}_{3}-10 / \mathrm{H}_{2}-3, \mathrm{H}_{2}-5$, and between $\mathrm{H}_{2}-9 / \mathrm{H}$ 5 eq). ${ }^{1)}$

Glucoside $11\left(\mathrm{C}_{16} \mathrm{H}_{30} \mathrm{O}_{9}, \mathrm{mp} 137-138^{\circ} \mathrm{C},[\alpha]_{\mathrm{D}}^{24}+22^{\circ}\right)$ and $12\left(\mathrm{C}_{16} \mathrm{H}_{30} \mathrm{O}_{9}\right.$, an amorphous powder, $\left.[\alpha]_{\mathrm{D}}^{24}+17^{\circ}\right)$ showed an $[\mathrm{M}+\mathrm{H}]^{+}$ion peak at $m / z \quad 367$ and an $[\mathrm{M}-$ $\left.\mathrm{C}_{6} \mathrm{H}_{12} \mathrm{O}_{6}+\mathrm{H}\right]^{+}$ion peak at $m / z 187$ in the positive FAB-MS. They were hydrolyzed with $\beta$-glucosidase and 5 from 11, and 6 from 12, were obtained from the hydrolyzed mixtures together with D-glucose. Consequently, 11 and 12 were monoglucosides of $\mathbf{5}$ and $\mathbf{6}$, respectively. The position of the $\beta$-glucosyl unit of both glucosides was confirmed to be C-2 from the HMBC correlation of glucosyl H-1/C-2 of 11, and from the observed NOE interaction between the glucosyl $\mathrm{H}-$ 1/H-2 in their NOESY spectra (Fig. 1). The absolute configurations at C-2 of $\mathbf{1 1}$ and $\mathbf{1 2}$ were indicated to be $S$ by the values of the glycosylation shift of the $\alpha$ - and $\beta$-carbons, and 
Table 2. ${ }^{1} \mathrm{H}-\mathrm{NMR}$ Chemical Shifts of $\mathbf{2}-\mathbf{1 5}$ (in Pyridine- $d_{5}, 500 \mathrm{MHz}$ )

\begin{tabular}{|c|c|c|c|c|}
\hline & 2 & 3 & & 4 \\
\hline $\mathrm{H}-3_{\mathrm{ax}}$ & $2.58 \mathrm{dd}(14.0,14.0)$ & $2.63 \mathrm{dd}(13.0,13.0)$ & $\mathrm{H}-1_{\mathrm{eq}}$ & $2.86 \mathrm{~m}$ \\
\hline $\mathrm{H}-3_{\mathrm{eq}}^{\mathrm{ax}}$ & $2.93 \mathrm{ddd}(2.0,2.0,14.0)$ & $3.12 \mathrm{dd}(2.0,13.0)$ & $\mathrm{H}-2_{\mathrm{ax}}^{\mathrm{c}}$ & 4.21 ddd $(3.5,3.5,12.0)$ \\
\hline $\mathrm{H}-4_{\mathrm{ax}}$ & $2.63 \mathrm{~m}$ & $2.65 \mathrm{~m}$ & $\mathrm{H}-3_{\mathrm{ax}}$ & 2.05 ddd $(12.0,12.0,12.0)$ \\
\hline $\mathrm{H}-5_{\mathrm{ax}}$ & $2.50 \mathrm{~m}$ & $2.39 \mathrm{~m}$ & $\mathrm{H}-3_{\mathrm{eq}}$ & 2.49 ddd $(3.5,3.5,12.0)$ \\
\hline $\mathrm{H}-5_{\mathrm{eq}}$ & 2.66 dddd $(2.0,3.0,5.5,13.0)$ & 2.47 ddd $(3.5,5.0,13.5)$ & $\mathrm{H}-4_{\mathrm{ax}}$ & 2.18 dddd $(3.5,3.5,12.0,12.0)$ \\
\hline H-6 & $6.65 \mathrm{brd}(5.5)$ & 6.62 brd $(5.0)$ & $\mathrm{H}-5_{\mathrm{ax}}^{\mathrm{ax}}$ & $1.93 \mathrm{ddd}(12.0,12.0,12.0)$ \\
\hline $\mathrm{H}_{3}-7$ & $1.83 \mathrm{~s}$ & $1.83 \mathrm{~s}$ & $\mathrm{H}-5_{\mathrm{eq}}$ & 2.25 ddd $(3.5,3.5,12.0)$ \\
\hline \multirow[t]{2}{*}{$\mathrm{H}_{2}-9$} & $3.89 \mathrm{~d}(11.0)$ & $3.87 \mathrm{~d}(11.0)$ & $\mathrm{H}-6_{\mathrm{ax}}^{\mathrm{q}}$ & $4.21 \mathrm{ddd}(3.5,3.5,12.0)$ \\
\hline & $3.92 \mathrm{~d}(11.0)$ & $3.92 \mathrm{~d}(11.0)$ & $\mathrm{H}_{3}-7$ & $1.50 \mathrm{~d}(7.0)$ \\
\hline \multirow[t]{3}{*}{$\mathrm{H}_{3}-10$} & $1.42 \mathrm{~s}$ & $1.41 \mathrm{~s}$ & $\mathrm{H}_{2}-9$ & $3.96 \mathrm{~d}(11.0)$ \\
\hline & & & & $3.99 \mathrm{~d}(11.0)$ \\
\hline & & & $\mathrm{H}_{3}-10$ & $1.47 \mathrm{~s}$ \\
\hline
\end{tabular}

\begin{tabular}{|c|c|c|c|c|}
\hline & 5 & 6 & 7 & 8 \\
\hline $\mathrm{H}-2_{\mathrm{ax}}$ & - & - & $3.71 \mathrm{dd}(3.0,12.0)$ & $3.73 \mathrm{dd}(4.0,12.0)$ \\
\hline $\mathrm{H}-\mathrm{e}_{\mathrm{eq}}$ & $4.24 \mathrm{dd}(3.0,3.0)$ & $4.27 \mathrm{dd}(3.0,3.0)$ & - & - \\
\hline $\mathrm{H}-3_{\mathrm{ax}}$ & $2.53 \mathrm{ddd}(3.0,13.0,13.0)$ & 2.62 ddd $(3.0,13.0,13.0)$ & $2.10 \operatorname{ddd}(12.0,12.0,12.0)$ & $2.25 \mathrm{ddd}(12.0,12.0,12.0)$ \\
\hline $\mathrm{H}-3_{\mathrm{eq}}$ & $2.27 \mathrm{ddd}(3.0,3.0,13.0)$ & 2.53 dddd $(3.0,3.0,3.0,13.0)$ & 2.33 ddd $(3.0,3.0,12.0)$ & 2.60 dddd $(3.0,3.0,4.0,12.0)$ \\
\hline $\mathrm{H}-4_{\mathrm{ax}}$ & 2.74 dddd $(3.0,3.0,13.0,13.0)$ & 2.76 dddd $(3.0,3.0,13.0,13.0)$ & 2.15 dddd $(3.0,3.0,12.0,12.0)$ & 2.12 dddd $(3.0,3.0,12.0,12.0)$ \\
\hline $\mathrm{H}-5_{\mathrm{ax}}$ & 2.28 dddd $(3.0,13.0,13.0,13.0)$ & 2.15 dddd $(3.0,13.0,13.0,13.0)$ & 2.10 br ddd $(12.0,12.0,12.0)$ & 1.97 dddd $(3.0,12.0,12.0,12.0)$ \\
\hline $\mathrm{H}-5_{\mathrm{eq}}$ & 2.19 dddd $(3.0,3.0,3.0,13.0)$ & 1.90 dddd $(3.0,3.0,3.0,13.0)$ & 2.07 dddd $(3.0,3.0,3.0,12.0)$ & 1.77 dddd $(3.0,3.0,3.0,12.0)$ \\
\hline$H-6_{a x}$ & $2.31 \mathrm{ddd}(3.0,13.0,13.0)$ & $2.26 \operatorname{ddd}(3.0,13.0,13.0)$ & 1.47 ddd $(3.0,12.0,12.0)$ & $1.47 \mathrm{ddd}(3.0,12.0,12.0)$ \\
\hline$H-6_{e q}$ & 1.92 ddd $(3.0,3.0,13.0)$ & $1.91 \mathrm{ddd}(3.0,3.0,13.0)$ & $2.11 \mathrm{ddd}(3.0,3.0,12.0)$ & $2.07 \mathrm{ddd}(3.0,3.0,12.0)$ \\
\hline $\mathrm{H}_{3}-7$ & $1.71 \mathrm{~s}$ & $1.71 \mathrm{~s}$ & $1.55 \mathrm{~s}$ & $1.55 \mathrm{~s}$ \\
\hline \multirow[t]{2}{*}{$\mathrm{H}_{2}-9$} & $3.97 \mathrm{~d}(11.0)$ & $3.94 \mathrm{~d}(10.5)$ & $3.92 \mathrm{~d}(10.5)$ & $3.92 \mathrm{~d}(10.5)$ \\
\hline & $4.02 \mathrm{~d}(11.0)$ & $4.02 \mathrm{~d}(10.5)$ & $3.99 \mathrm{~d}(10.5)$ & $3.97 \mathrm{~d}(10.5)$ \\
\hline $\mathrm{H}_{3}-10$ & $1.52 \mathrm{~s}$ & $1.49 \mathrm{~s}$ & $1.45 \mathrm{~s}$ & $1.44 \mathrm{~s}$ \\
\hline
\end{tabular}

9

\begin{tabular}{ll}
\hline $\mathrm{H}-2_{\mathrm{ax}}$ & $4.10 \mathrm{dd}(4.5,11.5)$ \\
$\mathrm{H}-2_{\mathrm{eq}}$ & - \\
$\mathrm{H}-3_{\mathrm{ax}}$ & $1.81 \mathrm{ddd}(11.5,12.0,12.0)$ \\
$\mathrm{H}-3_{\mathrm{eq}}$ & $2.47 \mathrm{ddd}(3.5,4.5,12.0)$ \\
$\mathrm{H}-4_{\mathrm{ax}}$ & $2.20 \mathrm{dddd}(3.5,3.5,12.0,12.0)$ \\
$\mathrm{H}-5_{\mathrm{ax}}$ & $1.68 \mathrm{dddd}(3.5,12.0,12.0,12.0)$ \\
$\mathrm{H}-5_{\mathrm{eq}}$ & $2.18 \mathrm{~m}$ \\
$\mathrm{H}-6_{\mathrm{ax}}$ & $1.85 \mathrm{ddd}(3.5,12.0,12.0)$ \\
$\mathrm{H}-6_{\mathrm{eq}}$ & $2.10 \mathrm{ddd}(3.5,3.5,12.0)$ \\
$\mathrm{H}_{3}-7$ & $1.60 \mathrm{~s}$ \\
$\mathrm{H}-9 \mathrm{a}$ & $3.93 \mathrm{~d}(11.0)$ \\
$\mathrm{H}-9 \mathrm{~b}$ & $3.96 \mathrm{~d}(11.0)$ \\
$\mathrm{H}_{3}-10$ & $1.46 \mathrm{~s}$ \\
$\mathrm{Glc}-1$ & -
\end{tabular}

10

$4.20 \mathrm{dd}(3.0,3.0)$

2.39 ddd $(3.0,13.0,13.0)$

2.32 ddd $(3.0,3.0,13.0)$

2.67 dddd $(3.0,3.0,13.0,13.0)$

2.01 dddd $(3.0,13.0,13.0,13.0)$

1.59 dddd $(3.0,3.0,3.0,13.0)$

2.05 ddd $(3.0,13.0,13.0)$

1.79 ddd $(3.0,3.0,13.0)$

$1.82 \mathrm{~s}$

$4.74 \mathrm{brs}$

$4.84 \mathrm{br} \mathrm{s}$

$1.73 \mathrm{~s}$

$5.02 \mathrm{~d}(7.5)$

11

\section{$4.17 \mathrm{dd}(3.0,3.0)$}

2.38 ddd $(3.0,13.0,13.0)$

2.69 ddd $(3.0,3.0,13.0)$

2.72 dddd $(3.0,3.0,13.0,13.0)$

2.19 dddd $(3.0,13.0,13.0,13.0)$

2.05 dddd $(3.0,3.0,3.0,13.0)$

2.10 ddd $(3.0,13.0,13.0)$

1.86 ddd $(3.0,3.0,13.0)$

$1.83 \mathrm{~s}$

$3.90 \mathrm{~d}(11.0)$

$4.06 \mathrm{~d}(11.0)$

$1.45 \mathrm{~s}$

$5.01 \mathrm{~d}(8.0)$

12

$-$

$4.21 \mathrm{dd}(3.0,3.0)$

2.48 ddd $(3.0,13.0,13.0)$

2.88 ddd $(3.0,3.0,13.0)$

2.75 dddd $(3.0,3.0,13.0,13.0)$

2.04 dddd $(3.0,13.0,13.0,13.0)$

1.74 dddd $(3.0,3.0,3.0,13.0)$

2.10 ddd $(3.0,13.0,13.0)$

1.81 ddd $(3.0,3.0,13.0)$

$1.81 \mathrm{~s}$

$3.92 \mathrm{~d}(11.0)$

$3.94 \mathrm{~d}(11.0)$

$1.42 \mathrm{~s}$

$5.00 \mathrm{~d}(8.0)$

\begin{tabular}{lllll}
\hline $\mathrm{H}-1_{\mathrm{eq}}$ & - & $2.45 \mathrm{~m}$ & $\mathrm{H}-2_{\mathrm{eq}}$ & $4.80 \mathrm{dd}(4.0,4.0)$ \\
$\mathrm{H}-2_{\mathrm{ax}}$ & $4.03 \mathrm{dd}(3.5,11.5)$ & $4.26 \mathrm{dd}(4.5,4.5,12.0)$ & $\mathrm{H}-3_{\mathrm{ax}}$ & $1.65 \mathrm{ddd}(4.0,12.0,12.0)$ \\
$\mathrm{H}-3_{\mathrm{ax}}$ & $1.71 \mathrm{ddd}(11.5,12.5,12.5)$ & $1.84 \mathrm{ddd}(12.0,12.0,12.0)$ & $\mathrm{H}_{\text {eq }}$ & $2.18 \mathrm{ddd}(4.0,4.0,12.0)$ \\
$\mathrm{H}-3_{\mathrm{eq}}$ & $2.45 \mathrm{ddd}(3.0,3.5,12.5)$ & $2.14 \mathrm{ddd}(3.0,4.5,12.0)$ & $\mathrm{H}-4_{\mathrm{ax}}$ & $2.81 \mathrm{dddd}(4.0,4.0,12.0,12.0)$ \\
$\mathrm{H}_{\mathrm{ax}}$ & $2.16 \mathrm{dddd}(3.0,3.0,12.5,12.5)$ & $2.11 \mathrm{dddd}(3.0,3.0,12.0,12.0)$ & $\mathrm{H}-5_{\mathrm{ax}}$ & $1.91 \mathrm{brdd}(12.0,17.5)$ \\
$\mathrm{H}-5_{\mathrm{ax}}$ & $1.60 \mathrm{dddd}(3.0,12.5,13.0,13.0)$ & $1.43 \mathrm{dddd}(3.0,12.0,12.0,12.0)$ & $\mathrm{H}-5_{\mathrm{eq}}$ & $2.15 \mathrm{ddd}(3.0,4.0,17.5)$ \\
$\mathrm{H}-5_{\mathrm{eq}}$ & $2.09 \mathrm{dddd}(3.0,3.0,3.0,13.0)$ & $1.51 \mathrm{dddd}(3.0,3.0,3.0,12.0)$ & $\mathrm{H}-6$ & $6.01 \mathrm{brd}(3.0)$ \\
$\mathrm{H}-6_{\mathrm{ax}}$ & $1.79 \mathrm{ddd}(3.0,13.0,13.0)$ & $1.44 \mathrm{dddd}(3.0,3.0,12.0,12.0)$ & $\mathrm{H}-7 \mathrm{a}$ & $4.55 \mathrm{~d}(12.0)$ \\
$\mathrm{H}-6_{\mathrm{eq}}$ & $2.05 \mathrm{ddd}(3.0,3.0,13.0)$ & $1.56 \mathrm{drddd}(3.0,3.0,12.0)$ & $\mathrm{H}-7 \mathrm{~b}$ & $4.97 \mathrm{~d}(12.0)$ \\
$\mathrm{H}_{3}-7$ & $1.57 \mathrm{~s}$ & $1.18 \mathrm{~d}(7.0)$ & $\mathrm{H}-9 \mathrm{a}$ & $4.78 \mathrm{br} \mathrm{s}$ \\
$\mathrm{H}-9 \mathrm{a}$ & $3.84 \mathrm{~d}(10.5)$ & $5.00 \mathrm{~d}(2.0)$ & $\mathrm{H}-9 \mathrm{~b}$ & $4.80 \mathrm{brs}$ \\
$\mathrm{H}-9 \mathrm{~b}$ & $4.29 \mathrm{~d}(10.5)$ & $5.28 \mathrm{~d}(2.0)$ & $\mathrm{H}_{3}-10$ & $1.68 \mathrm{~s}$ \\
$\mathrm{H}_{3}-10$ & $1.37 \mathrm{~s}$ & - & $\mathrm{Glc}-1$ & $4.98 \mathrm{~d}(7.5)$ \\
$\mathrm{H}_{2}-10$ & - & $4.36 \mathrm{br} \mathrm{s}$ & & \\
$\mathrm{Glc}-1$ & $4.96 \mathrm{~d}(8.0)$ & $5.02 \mathrm{~d}(7.5)$ & &
\end{tabular}


<smiles>C=C(C)[C@@H]1CC[C@](C)(O)[C@H](O)C1</smiles>

1

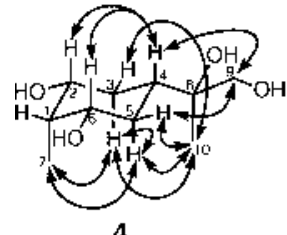

4

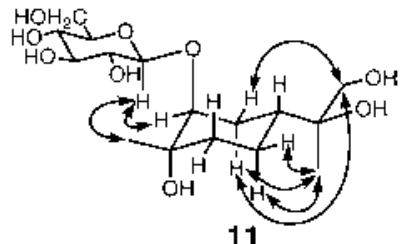

11

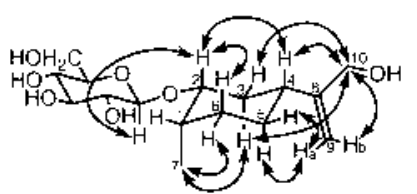

14

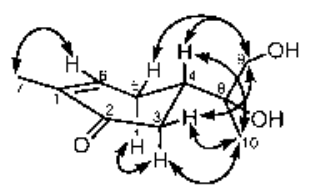

2

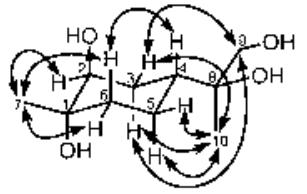

5

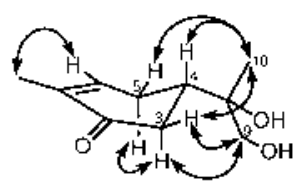

3

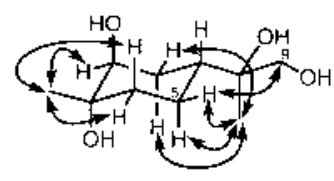

6

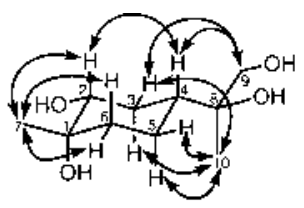

7

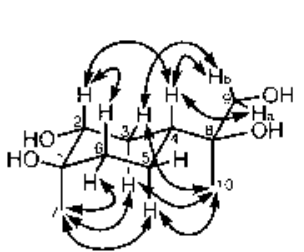

9

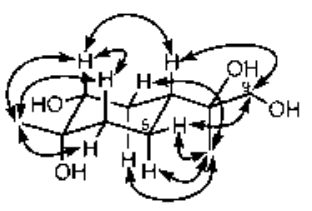

8

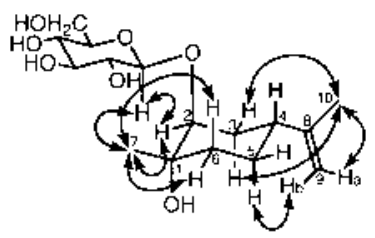

10

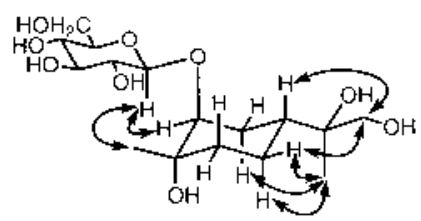

12

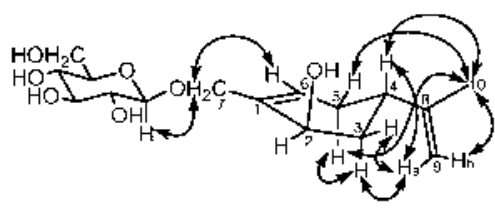

15

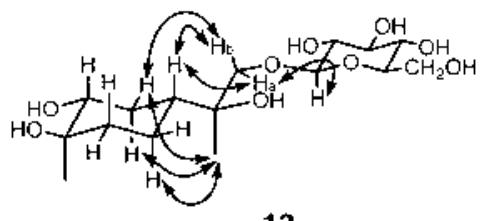

13

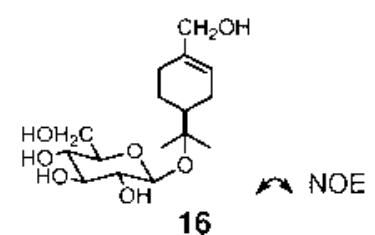

Fig. 1. Structures of $\mathbf{1}-\mathbf{1 6}$, and NOE Interactions Observed in the NOESY Spectra of $\mathbf{2}-\mathbf{1 5}$

the chemical shift of the glucosyl anomeric carbon, as shown in Table $1^{1,8)}$ Thus $\mathbf{5}, \mathbf{6}, \mathbf{1 1}$, and $\mathbf{1 2}$ were characterized as $(1 S, 2 S, 4 R, 8 S)$ - $p$-menthane-1,2,8,9-tetrol, (1S,2S,4R,8R)- $p$ menthane-1,2,8,9-tetrol, $(1 S, 2 S, 4 R, 8 S)$ - $p$-menthane-1,2,8,9tetrol $2-O-\beta$-D-glucopyranoside, and $(1 S, 2 S, 4 R, 8 R)-p$-menthane-1,2,8,9-tetrol 2- $O$ - $\beta$-D-glucopyranoside, respectively.

Tetrols $7\left(\mathrm{C}_{10} \mathrm{H}_{20} \mathrm{O}_{4}\right.$, an amorphous powder, $\left.[\alpha]_{\mathrm{D}}^{23}+11^{\circ}\right)$ and $8\left(\mathrm{C}_{10} \mathrm{H}_{20} \mathrm{O}_{4}\right.$, an amorphous powder, $\left.[\alpha]_{\mathrm{D}}^{23}+26^{\circ}\right)$ showed similar ${ }^{1} \mathrm{H}$ - and ${ }^{13} \mathrm{C}$-NMR spectral features (Tables 2,1 ) and revealed an $[\mathrm{M}+\mathrm{H}]^{+}$ion peak at $m / z 205$ in the positive FAB-MS. From the result of the HMBC experiment on 7 (H$4_{\mathrm{ax}} / \mathrm{C}-3, \mathrm{C}-5, \mathrm{C}-6, \mathrm{C}-8, \mathrm{C}-9 ; \mathrm{H}_{3}-7 / \mathrm{C}-1, \mathrm{C}-2, \mathrm{C}-6$; $\mathrm{H}_{2}-9 / \mathrm{C}-4$, $\left.\mathrm{C}-8, \mathrm{C}-10 ; \mathrm{H}_{3}-10 / \mathrm{C}-4, \mathrm{C}-8, \mathrm{C}-9\right)$, they were also indicated to be $p$-menthane-1,2,8,9-tetrol. The configuration of the $\mathrm{C}-2$ hydroxyl was concluded to be equatorial by the axial $\mathrm{H}-2$ signal pattern of their ${ }^{1} \mathrm{H}-\mathrm{NMR}$ spectra $(\mathrm{dd}, J=3.0,12.0 \mathrm{~Hz}$ for 7 and dd, $J=4.0,12.0 \mathrm{~Hz}$ for 8; Table 2). The stereochemical relationship between $\mathrm{C}-7$ and $\mathrm{C}-8$ was suggested to be trans from the observed NOE interactions in their NOESY spectra (Fig. 1). Therefore 7 and $\mathbf{8}$ were concluded to be stereoisomers at the C-8 of trans- $p$-menthane- $1,2_{\mathrm{eq}}, 8,9$-tetrol, respectively. The ${ }^{13} \mathrm{C}$ chemical shifts of $\mathrm{C}-3(\mathbf{7}, \delta 33.0 ; 8, \delta$ $31.9)$ and $\mathrm{C}-5(7, \delta 22.1 ; \mathbf{8}, \delta 23.1)$ suggested that the relative configurations at C-4 and C- 8 were $4 R^{*}, 8 S^{*}$ for 7 and $4 R^{*}, 8 R^{*}$ for 8 . This suggestion was supported by the NOESY spectrum of 7, which showed NOE interactions between $\mathrm{H}_{3}-10 / \mathrm{H}-3_{\mathrm{ax}}, \mathrm{H}_{2}-5$, and between $\mathrm{H}_{2}-9 / \mathrm{H}-3_{\text {eq }}$, and that of 8 which showed NOE interactions between $\mathrm{H}_{3}-10 / \mathrm{H}_{2}-3$, $\mathrm{H}_{2}-5$, and between $\mathrm{H}_{2}-9 / \mathrm{H}-5_{\text {eq }}$ (Fig. 1). Therefore 7 and 8 were concluded to be rel-( $1 S, 2 R, 4 R, 8 S)$ - $p$-menthane-1,2,8,9tetrol and rel-( $1 S, 2 R, 4 R, 8 R)-p$-menthane-1,2,8,9-tetrol, respectively.

Tetrol $9\left(\mathrm{C}_{10} \mathrm{H}_{20} \mathrm{O}_{4}\right.$, an amorphous powder, $\left.[\alpha]_{D}^{21}-3^{\circ}\right)$ was indicated to be $p$-menthane-1,2,8,9-tetrol by the same methods as described in $\mathbf{2}$ to $\mathbf{8}$, and the configuration of the C-2 hydroxyl was concluded to be equatorial by the axial $\mathrm{H}-2$ signal patterns of the ${ }^{1} \mathrm{H}-\mathrm{NMR}$ spectrum (Table 1 ). The stereochemical relationship between $\mathrm{C}-7$ and $\mathrm{C}-8$ was suggested to be $c i s$ from the observed NOE interactions between $\mathrm{H}_{3}-7 / \mathrm{H}-3_{\mathrm{ax}}, \mathrm{H}-5_{\mathrm{ax}}$, and between $\mathrm{H}-2_{\mathrm{ax}} / \mathrm{H}-4_{\mathrm{ax}}, \mathrm{H}-6_{\mathrm{ax}}$ in the NOESY spectra, and comparison of chemical shifts of $\mathrm{H}-3_{\mathrm{ax}}$ $(\delta 1.81)$ and $\mathrm{H}-5_{\mathrm{ax}}(\delta 1.68)$ with those of 7 and 8 , which were lowfield shifted by the effect of the C-1 axial hydroxyl group $\left(\mathrm{H}-3_{\mathrm{ax}}, 7 ; \delta 2.10,8 ; \delta 2.10 ; \mathrm{H}-5_{\mathrm{ax}}, 7 ; \delta 2.25,8 ; \delta\right.$ 1.97). Therefore 9 was revealed to be $c i s-p$-menthane$1,2_{\text {eq }}, 8,9$-tetrol. In addition, the NOESY spectrum of 9 showed the NOE interactions between $\mathrm{H}_{3}-10 / \mathrm{H}_{2}-3, \mathrm{H}-5_{\mathrm{ax}}$, and between $\mathrm{H}-9 \mathrm{~b} / \mathrm{H}-3_{\text {eq }}$, which were also observed in the NOESY spectra of 4 and 7 (Fig. 1). The relative configuration at C-4 and C-8 was assumed to be $4 R^{*}, 8 S^{*}$. From this evidence, 9 was concluded to be rel-( $1 R, 2 R, 4 R, 8 S)-p$-menthane-1,2,8,9-tetrol.

Glucoside $13\left(\mathrm{C}_{16} \mathrm{H}_{30} \mathrm{O}_{9}\right.$, an amorphous powder, $[\alpha]_{\mathrm{D}}^{23}$ $-15^{\circ}$ ) showed an $[\mathrm{M}+\mathrm{H}]^{+}$ion peak at $\mathrm{m} / z 367$ and an $\left[\mathrm{M}-\mathrm{C}_{6} \mathrm{H}_{12} \mathrm{O}_{6}+\mathrm{H}\right]^{+}$ion peak at $m / z 187$ in the positive FABMS. By comparison of ${ }^{1} \mathrm{H}$ - and ${ }^{13} \mathrm{C}-\mathrm{NMR}$ data with those of 9 (Tables 2, 1), and from the observed NOE interaction between the glucosyl H-1/H-9b in the NOESY spectrum (Fig. 
1), 13 was suggested to be a glucoside of 9 , and the position of attachment of the glucosyl unit was revealed to be C-9. Since the glucose was suggested to be D-form from its $[M]_{D}$ value $\left[\Delta[M]_{\mathrm{D}}(\mathbf{1 3}-\mathbf{9})-47^{\circ}\right.$; methyl $\beta$-D-glucopyranoside $\left.-62^{\circ}\right],{ }^{4)} 13$ was concluded to be rel- $(1 R, 2 R, 4 R, 8 S)$ - $p$-menthane-1,2,8,9-tetrol 9- $O-\beta$-D-glucopyranoside.

Glucoside $14\left(\mathrm{C}_{16} \mathrm{H}_{28} \mathrm{O}_{7}\right.$, an amorphous powder, $[\alpha]_{D}^{25}$ $-26^{\circ}$ ) showed an $[\mathrm{M}+\mathrm{H}]^{+}$ion peak at $\mathrm{m} / \mathrm{z} 333$ and an $\left[\mathrm{M}-\mathrm{C}_{6} \mathrm{H}_{12} \mathrm{O}_{6}+\mathrm{H}\right]^{+}$ion peak at $\mathrm{m} / z 153$ in the positive FABMS. The ${ }^{1} \mathrm{H}-,{ }^{13} \mathrm{C}$-, and ${ }^{13} \mathrm{C}-{ }^{1} \mathrm{H}$ correlation spectroscopy (COSY) NMR spectral data (Tables 2, 1) showed the presence of one $\beta$-glucopyranosyl, one sec-methyl, four methylenes (one oxygenated), three methines (one oxygenated), and one terminal-methylene group. From the result of the HMBC experiment on $14\left(\mathrm{H}-4_{\text {ax }} / \mathrm{C}-2, \mathrm{C}-5, \mathrm{C}-6, \mathrm{C}-8, \mathrm{C}-9, \mathrm{C}-10 ; \mathrm{H}_{3}-\right.$ 7/C-1, C-6; H-9a/C-4, C-8, C-10; H-9b/C-4, C-8, C-10; $\mathrm{H}_{3}-$ 10/C-4, C-8, C-9; Glc H-1/C-2), the aglycone was suggested to be $p$-menth-8-ene-2,10-diol and the position of the glucosyl unit was found to be C-2. The configuration of C-2 hydroxyl was concluded to be equatorial by the axial H-2 signal pattern of its ${ }^{1} \mathrm{H}-\mathrm{NMR}$ spectrum (ddd, $J=4.5,4.5,12.0 \mathrm{~Hz}$; Table 2). The stereochemical relationship between $\mathrm{C}-7$ and C-8 was indicated to be cis, and the conformation of the cyclohexane ring was suggested to be the chair form from the NOE interactions between $\mathrm{H}_{3}-7 / \mathrm{H}-3_{\mathrm{ax}}, \mathrm{H}-6_{\mathrm{eq}}$ and between $\mathrm{H}-$ $2_{\mathrm{ax}} / \mathrm{H}-4_{\mathrm{ax}}, \mathrm{H}-6_{\mathrm{ax}}$ in the NOESY spectrum (Fig. 1). Enzymatic hydrolysis of $\mathbf{1 4}$ gave an aglycone (an amorphous powder, $\left.[\alpha]_{\mathrm{D}}^{22}-11^{\circ} ; 14 \mathrm{a}\right)$ and D-glucose, and the absolute configuration at C-2 was confirmed to be $R$ by the values of the glycosylation shift of the $\alpha$ - and $\beta$-carbons, and the chemical shift of the glucosyl anomeric carbon, as shown in Table 1.,8) From these data, 14 was characterized as $(1 S, 2 R, 4 R)-p$ menth-8-ene-2,10-diol 2- $O$ - $\beta$-D-glucopyranoside.

Glucoside $15\left(\mathrm{C}_{16} \mathrm{H}_{26} \mathrm{O}_{7}\right.$, an amorphous powder, $[\alpha]_{D}^{24}$ $\left.+12^{\circ}\right)$ showed an $[\mathrm{M}+\mathrm{H}]^{+}$ion peaks at $m / z 331$ and an $\left[\mathrm{M}-\mathrm{C}_{6} \mathrm{H}_{12} \mathrm{O}_{6}+\mathrm{H}\right]^{+}$ion peaks at $\mathrm{m} / \mathrm{z} 151$ in the positive FAB-MS. Enzymatic hydrolysis of $\mathbf{1 5}$ gave an aglycone (an amorphous powder, $\left.[\alpha]_{\mathrm{D}}^{22}+36^{\circ} ; \mathbf{1 5 a}\right)$ and D-glucose. The ${ }^{1} \mathrm{H}$, ${ }^{13} \mathrm{C}$-, and ${ }^{13} \mathrm{C}-{ }^{1} \mathrm{H}$ COSY NMR spectral data (Tables 2,1 ) of 15 showed the presence of one $\beta$-glucopyranosyl, one tertmethyl, three methylenes (one oxygenated), two methines (one oxygenated), one tri-substituted double bond, and one terminal-methylene group, and the result of the HMBC experiment $\left(\mathrm{H}-6 / \mathrm{C}-2, \mathrm{C}-4, \mathrm{C}-5, \mathrm{C}-7\right.$; $\mathrm{H}_{2}-7 / \mathrm{C}-1, \mathrm{C}-2$, C-6, Glc C-1; H-9a/C-4, C-8, C-10; H-9b/C-4, C-8, C-10; $\mathrm{H}_{3}-10 / \mathrm{C}-4$, $\mathrm{C}-8$, C-9; Glc H-1/C-7) suggested that 15 was 7-hydroxycarveol $7-O-\beta$-D-glucopyranoside. As the equatorial $\mathrm{H}-2$ signal pattern was shown in the ${ }^{1} \mathrm{H}-\mathrm{NMR}$ spectrum (dd, $J=4.0$, 4.0 Hz; Table 2), and the NOE interactions between $\mathrm{H}-3_{\mathrm{ax}} / \mathrm{H}-$ $5_{\mathrm{ax}}$, between $\mathrm{H}-\mathrm{9a} / \mathrm{H}-3_{\mathrm{ax}}, \mathrm{H}-3_{\text {eq }}, \mathrm{H}-4$, and between $\mathrm{H}_{3}-10 / \mathrm{H}-4$, $\mathrm{H}-5_{\mathrm{ax}}, \mathrm{H}-5_{\mathrm{eq}}$ were observed in the NOESY spectrum (Fig. 1), the configuration of the C-2 hydroxyl was concluded to be axial and the stereochemical relationship between the C-2 hydroxyl and C-4 isopropenyl group was trans. It has been reported that the $(2 R, 4 S)$ - and $(2 S, 4 R)$-forms of carveol show positive and negative optical rotations, respectively. $\left.{ }^{9}\right) \mathrm{Be}-$ couse 15a showed positive optical rotation, 15 was concluded to be $(2 R, 4 S)$-7-hydroxycarveol 7-O- $\beta$-D-glucopyranoside.

\section{Experimental}

Melting points were determined on a Yanagimoto micromelting point apparatus and are uncorrected. Optical rotations were measured on a JASCO DIP-370 digital polarimeter. The CD spectra were recorded with a JASCO J600 spectropolarimeter at $23^{\circ} \mathrm{C}$. FAB-MS were recorded with a JEOL HX110 spectrometer using glycerol as a matrix. ${ }^{1} \mathrm{H}$ - and ${ }^{13} \mathrm{C}$-NMR spectra were recorded on JEOL A-500 spectrometers with TMS as an internal standard, and chemical shifts were recorded as $\delta$ values. ${ }^{13} \mathrm{C}-{ }^{1} \mathrm{H}$ COSY, HMBC, NOESY, and 1D-NOESY spectra were obtained with the usual pulse sequence, and data processing was performed with standard JEOL software. Column chromatography was carried out under TLC monitoring using Kieselgel 60 (70-230 mesh, Merck), Sephadex LH-20 (25-100 $\mu \mathrm{m}$, Pharmacia), Lobar RP-8 column (Merck), and Amberlite XAD-II (Organo). TLC was performed on silica gel (Merck 5721), and spots were detected with $p$ anisaldehyde- $\mathrm{H}_{2} \mathrm{SO}_{4}$ reagent. HPLC separation was carried out with Symmetryprep $\mathrm{C}_{18} 7 \mu \mathrm{m}$ (Waters; column size, $7.8 \times 300 \mathrm{~mm}$; octadecyl silica (ODS)), Carbohydrate Analysis (Waters; column size, $3.9 \times 300 \mathrm{~mm}$; CHA) columns. Acetylation was done in the usual way using $\mathrm{Ac}_{2} \mathrm{O}$ and pyridine. No acetoxyl group had been detected by NMR spectral analysis of the materials prior to acetylation.

Extraction and Separation Commercial caraway (the fruit of Carum carvi L.; purchased from Asaoka Spices Ltd., lot no. 93010, $2.0 \mathrm{~kg}$ ) was extracted with $70 \%$ methanol $(41 \times 2)$, and the extract was partitioned into ether-water and ethyl acetate-water, respectively. The aqueous portion was chromatographed on Amberlite XAD-II $\left(\mathrm{H}_{2} \mathrm{O} \rightarrow \mathrm{MeOH}\right)$, and the methanol eluate $(27.6 \mathrm{~g})$ was subjected to Sephadex LH-20 (MeOH) column chromatography to give eight fractions (frs. $\mathrm{A}-\mathrm{H})$. Fraction $\mathrm{B}(18.9 \mathrm{~g})$ was chromatographed on silica gel $\left[\mathrm{CHCl}_{3}-\mathrm{MeOH}-\mathrm{H}_{2} \mathrm{O} \quad(17: 3: 0.2 \rightarrow\right.$ $4: 3: 0.1 \rightarrow 7: 3: 0.5) \rightarrow \mathrm{MeOH}]$ to give 14 fractions (frs. $\mathrm{B}_{1}-\mathrm{B}_{14}$ ). Fraction $\mathrm{B}_{2}(1.32 \mathrm{~g})$ was passed through a Lobar RP-8 column $\left[\mathrm{MeCN}-\mathrm{H}_{2} \mathrm{O}\right.$ $(1: 4 \rightarrow 1: 1)$ ] to give seven fractions (frs. $\left.B_{2-1}-B_{2-7}\right)$, and fr. $B_{2-2}$ was subjected to HPLC [ODS, MeOH- $\left.\mathrm{H}_{2} \mathrm{O}(1: 4)\right]$ and silica gel $\left[\mathrm{CHCl}_{3}-\mathrm{MeOH}\right.$ (19:1)] chromatography to give $\mathbf{2}(7 \mathrm{mg})$ and $\mathbf{3}(9 \mathrm{mg})$. Fraction $\mathrm{B}_{2-4}$ was subjected to HPLC [ODS, $\mathrm{MeCN}-\mathrm{H}_{2} \mathrm{O}(3: 7)$ ] to give $1(4 \mathrm{mg})$. Fraction $\mathrm{B}_{5}$ $(0.44 \mathrm{~g})$ was subjected to a Lobar RP-8 column $\left[\mathrm{MeCN}-\mathrm{H}_{2} \mathrm{O}(3: 17)\right]$ and HPLC [CHA, MeCN- $\mathrm{H}_{2} \mathrm{O}(19: 1)$ ] to give $15(8 \mathrm{mg})$. Fraction $\mathrm{B}_{6}(0.94 \mathrm{~g})$ was passed through a Lobar RP-8 column $\left[\mathrm{MeCN}-\mathrm{H}_{2} \mathrm{O}(3: 17)\right]$ to give eight fractions (frs. $\mathrm{B}_{6-1}-\mathrm{B}_{6-8}$ ). Fraction $\mathrm{B}_{6-2}$ was subjected to HPLC [ODS, $\mathrm{MeCN}-\mathrm{H}_{2} \mathrm{O}(1: 19)$ ] to give $7(7 \mathrm{mg})$ and $8(5 \mathrm{mg})$. Fraction $\mathrm{B}_{6-5}$ was subjected to HPLC [CHA, MeCN- $\left.\mathrm{H}_{2} \mathrm{O}(39: 7)\right]$ and then ODS $\left[\mathrm{MeCN}-\mathrm{H}_{2} \mathrm{O}\right.$ (7:33)] to give $\mathbf{1 4}(11 \mathrm{mg})$. Fraction $\mathrm{B}_{6-6}$ was subjected to silica gel column chromatography $\left[\mathrm{CHCl}_{3}-\mathrm{MeOH}-\mathrm{H}_{2} \mathrm{O}(17: 3: 0.2)\right]$ and then HPLC [CHA, $\left.\mathrm{MeCN}-\mathrm{H}_{2} \mathrm{O}(97: 3)\right]$ to give $\mathbf{1 0}(302 \mathrm{mg})$. Fraction $\mathrm{B}_{7}(0.99 \mathrm{~g})$ was subjected to a Lobar RP-8 column [MeCN- $\mathrm{H}_{2} \mathrm{O}$ (3:17)] and Sephadex LH-20 $(\mathrm{MeOH})$ to give $16(38 \mathrm{mg})$. Fraction $\mathrm{B}_{8}(0.60 \mathrm{~g})$ was subjected to a Lobar RP-8 column [MeCN- $\left.\mathrm{H}_{2} \mathrm{O}(3: 17)\right]$ and HPLC [ODS, $\mathrm{MeCN}-\mathrm{H}_{2} \mathrm{O}(1: 19)$ ] to give $5(5 \mathrm{mg}), \mathbf{6}(16 \mathrm{mg})$, and $9(25 \mathrm{mg})$, respectively. Fraction $\mathrm{B}_{10}(1.38 \mathrm{~g})$ was also subjected to a Lobar RP-8 column $\left[\mathrm{MeCN}-\mathrm{H}_{2} \mathrm{O}(3: 17)\right]$ to give eight fractions (frs. $\mathrm{B}_{10-1}-\mathrm{B}_{10-8}$ ). Fraction $\mathrm{B}_{10-2}$ was subjected to silica gel column chromatography $\left[\mathrm{CHCl}_{3}-\mathrm{MeOH}-\mathrm{H}_{2} \mathrm{O}(8: 2: 0.2)\right]$ to give $4(98 \mathrm{mg})$. Fraction $\mathrm{B}_{14}(1.44 \mathrm{~g})$ was passed through a Lobar RP-8 column $\left[\mathrm{MeCN}-\mathrm{H}_{2} \mathrm{O}\right.$ (3:17)] to give eight fractions (frs. $B_{14-1}-B_{14-8}$ ), and fr. $B_{14-3}$ was acetylated with $\mathrm{Ac}_{2} \mathrm{O}$ and pyridine, and the acetylated fraction was subjected to silica gel column chromatography $\left[\mathrm{CHCl}_{3}-\mathrm{MeOH}(20: 1)\right]$ and then HPLC [ODS, $\left.\mathrm{MeCN}-\mathrm{H}_{2} \mathrm{O}(2: 3)\right]$ to give two fractions. These two fractions were deacetylated by heating in a water bath with $5 \% \mathrm{NH}_{4} \mathrm{OH}-\mathrm{MeOH}$ for $2 \mathrm{~h}$. From the former fraction, $\mathbf{1 1}(16 \mathrm{mg})$ and $\mathbf{1 3}(2 \mathrm{mg})$ were isolated by HPLC [CHA, $\left.\mathrm{MeCN}-\mathrm{H}_{2} \mathrm{O}(7: 13)\right]$, and from the latter fraction, $12(9 \mathrm{mg})$ was isolated by Sephadex LH-20 (MeOH) column chromatography.

The following compounds were identified by comparison with authentic compounds or published physical and spectral data: $(1 S, 2 S, 4 R)-p$-menth- 8 ene-1,2-diol (1) and ( $4 S)$ - $p$-menth-1-ene-7,8-diol 8- $O$ - $\beta$-D-glucopyranoside (16).

(4S,8R)-8,9-Dihydroxy-8,9-dihydrocarvone (2) An amorphous powder, $[\alpha]_{\mathrm{D}}^{25}-6^{\circ}(c=0.1, \mathrm{MeOH})$. Positive FAB-MS $m / z: 369[2 \mathrm{M}+\mathrm{H}]^{+}, 223$ $[\mathrm{M}+\mathrm{K}]^{+}, 207[\mathrm{M}+\mathrm{Na}]^{+}$(base), $185.1175[\mathrm{M}+\mathrm{H}]^{+}$(Calcd for $\mathrm{C}_{10} \mathrm{H}_{17} \mathrm{O}_{3}$ : 185.1177), $167\left[\mathrm{M}-\mathrm{H}_{2} \mathrm{O}+\mathrm{H}\right]^{+}, 149\left[\mathrm{M}-2 \mathrm{H}_{2} \mathrm{O}+\mathrm{H}\right]^{+}$. ${ }^{1} \mathrm{H}-\mathrm{NMR}$ (pyridine$\left.d_{5}, 500 \mathrm{MHz}\right) \delta$ : Table $2 .{ }^{13} \mathrm{C}-\mathrm{NMR}$ (pyridine- $\left.d_{5}, 125 \mathrm{MHz}\right) \delta$ : Table $1 . \mathrm{CD}$ : $(c=0.0100 \mathrm{M}, \mathrm{MeOH}) \Delta \varepsilon(\mathrm{nm}):-0.48(250)$.

(4S,8S)-8,9-Dihydroxy-8,9-dihydrocarvone (3) An amorphous powder, $[\alpha]_{\mathrm{D}}^{25}-7^{\circ}(c=0.2, \mathrm{MeOH})$. Positive FAB-MS $m / z: 369[2 \mathrm{M}+\mathrm{H}]^{+}, 223$ $[\mathrm{M}+\mathrm{K}]^{+}, 207[\mathrm{M}+\mathrm{Na}]^{+}$(base), $185.1180[\mathrm{M}+\mathrm{H}]^{+}$(Calcd for $\mathrm{C}_{10} \mathrm{H}_{17} \mathrm{O}_{3}$ : 185.1177), $167\left[\mathrm{M}-\mathrm{H}_{2} \mathrm{O}+\mathrm{H}\right]^{+}, 149\left[\mathrm{M}-2 \mathrm{H}_{2} \mathrm{O}+\mathrm{H}^{+}\right.$. ${ }^{1} \mathrm{H}-\mathrm{NMR}$ (pyridine$\left.d_{5}, 500 \mathrm{MHz}\right) \delta$ : Table $2 .{ }^{13} \mathrm{C}-\mathrm{NMR}$ (pyridine- $d_{5}, 125 \mathrm{MHz}$ ) $\delta$ : Table 1. CD: 
$(c=0.0125 \mathrm{M}, \mathrm{MeOH}) \Delta \varepsilon(\mathrm{nm}):-0.39(240) . \mathrm{HMBC}$ correlations: $\mathrm{H}-3_{\mathrm{ax}} / \mathrm{C}-$ 2, C-4, C-5; H-3 ${ }_{\mathrm{eg}} / \mathrm{C}-1, \mathrm{C}-2, \mathrm{C}-4, \mathrm{C}-5, \mathrm{C}-8 ; \mathrm{H}-4{ }_{\mathrm{ax}} / \mathrm{C}-2, \mathrm{C}-3, \mathrm{C}-5, \mathrm{C}-8, \mathrm{C}-9$, C-10; H-5 ax $/ \mathrm{C}-1, \mathrm{C}-3$, C-4, C-6; H-5 eq $/ \mathrm{C}-3$, C-4; H-6/C-4, C-5, C-7; H $-7 / \mathrm{C}-$ 1, C-2, C-6; $\mathrm{H}_{2}-9 / \mathrm{C}-4, \mathrm{C}-8, \mathrm{C}-10 ; \mathrm{H}_{3}-10 / \mathrm{C}-4, \mathrm{C}-8, \mathrm{C}-9$.

$4 \beta \mathrm{H}$-cis-p-Menthane-2 $\alpha, 6 \alpha, 8,9$-tetrol (4) An amorphous powder, $[\alpha]_{\mathrm{D}}^{21}+4^{\circ}(c=1.6, \mathrm{MeOH})$. Positive FAB-MS $m / z: 205.1448[\mathrm{M}+\mathrm{H}]^{+}$(base, Calcd for $\left.\mathrm{C}_{10} \mathrm{H}_{21} \mathrm{O}_{4}: 205.1439\right), 187\left[\mathrm{M}-\mathrm{H}_{2} \mathrm{O}+\mathrm{H}\right]^{+}, 169\left[\mathrm{M}-2 \mathrm{H}_{2} \mathrm{O}+\mathrm{H}\right]^{+}$. ${ }^{1} \mathrm{H}-\mathrm{NMR}$ (pyridine- $\left.d_{5}, 500 \mathrm{MHz}\right) \quad \delta$ : Table $2 .{ }^{13} \mathrm{C}-\mathrm{NMR}$ (pyridine- $d_{5}$, $125 \mathrm{MHz}) \delta$ : Table 1 . HMBC correlations: $\mathrm{H}-1_{\mathrm{eq}} / \mathrm{C}-2$, C-3, C-5, C-6, C-7; $\mathrm{H}-2_{\mathrm{ax}} / \mathrm{C}-1, \mathrm{C}-3, \mathrm{C}-4, \mathrm{C}-6, \mathrm{C}-7$; H-3 ${ }_{\mathrm{ax}} / \mathrm{C}-1, \mathrm{C}-2, \mathrm{C}-4, \mathrm{C}-5, \mathrm{C}-8$; H-3 ${ }_{\mathrm{eq}} / \mathrm{C}-1, \mathrm{C}-$ 2, C-4, C-5; H- 4 ax $/ \mathrm{C}-2$, C-3, C-5, C-6, C-8, C-9, C-10; H-5 ${ }_{\mathrm{ax}} / \mathrm{C}-1, \mathrm{C}-3, \mathrm{C}-4$, C-6, C-8; H-5 $/$ eq $/ \mathrm{C}-1, \mathrm{C}-3, \mathrm{C}-4, \mathrm{C}-6$; H-6 ${ }_{\mathrm{ax}} / \mathrm{C}-1, \mathrm{C}-2, \mathrm{C}-5, \mathrm{C}-7$; $\mathrm{H}_{3}-7 / \mathrm{C}-1, \mathrm{C}-$ 2, C-6; $\mathrm{H}_{2}-9 / \mathrm{C}-4, \mathrm{C}-8, \mathrm{C}-10 ; \mathrm{H}_{3}-10 / \mathrm{C}-4, \mathrm{C}-8$, C-9.

$(1 S, 2 S, 4 R, 8 S)-p$-Menthane-1,2,8,9-tetrol (5) An amorphous powder, $[\alpha]_{\mathrm{D}}^{22}+30^{\circ}(c=0.4, \mathrm{MeOH})$. Positive FAB-MS $m / z: 243[\mathrm{M}+\mathrm{K}]^{+}, 227.1245$ $[\mathrm{M}+\mathrm{Na}]^{+}\left(\right.$Calcd for $\left.\mathrm{C}_{10} \mathrm{H}_{20} \mathrm{O}_{4} \mathrm{Na}: 227.1259\right), 205[\mathrm{M}+\mathrm{H}]^{+}, 187[\mathrm{M}-$ $\left.\mathrm{H}_{2} \mathrm{O}+\mathrm{H}\right]^{+}, 169\left[\mathrm{M}-2 \mathrm{H}_{2} \mathrm{O}+\mathrm{H}\right]^{+}$(base). ${ }^{1} \mathrm{H}-\mathrm{NMR}$ (pyridine- $d_{5}, 500 \mathrm{MHz}$ ) $\delta$ : Table $2 .{ }^{13} \mathrm{C}-\mathrm{NMR}$ (pyridine- $\left.d_{5}, 125 \mathrm{MHz}\right) \delta$ : Table 1 . HMBC correlations: $\mathrm{H}-2{ }_{\mathrm{eq}} / \mathrm{C}-1, \mathrm{C}-4 ; \mathrm{H}-3_{\mathrm{ax}} / \mathrm{C}-4, \mathrm{C}-5, \mathrm{C}-8 ; \mathrm{H}-3$ eq $/ \mathrm{C}-5 ; \mathrm{H}-4_{\mathrm{ax}} / \mathrm{C}-5, \mathrm{C}-9 ; \mathrm{H}-$ $5{ }_{\mathrm{ax}} / \mathrm{C}-6 ; \mathrm{H}-5_{\mathrm{eq}} / \mathrm{C}-6 ; \mathrm{H}-6_{\mathrm{ax}} / \mathrm{C}-1, \mathrm{C}-2, \mathrm{C}-4, \mathrm{C}-7 ; \mathrm{H}-6_{\mathrm{eq}} / \mathrm{C}-1, \mathrm{C}-2, \mathrm{C}-5 ; \mathrm{H}_{3}-7 / \mathrm{C}-1$, C-2, C-6; H $\mathrm{H}_{2}-9 / \mathrm{C}-4, \mathrm{C}-8, \mathrm{C}-10 ; \mathrm{H}_{3}-10 / \mathrm{C}-4, \mathrm{C}-8, \mathrm{C}-9$.

$(1 R, 2 S, 4 R, 8 R)-p$-Menthane-1,2,8,9-tetrol (6) An amorphous powder, $[\alpha]_{\mathrm{D}}^{22}+27^{\circ}(c=0.8, \mathrm{MeOH})$. Positive FAB-MS $m / z: 243[\mathrm{M}+\mathrm{K}]^{+}, 227$ $[\mathrm{M}+\mathrm{Na}]^{+}, 205.1424[\mathrm{M}+\mathrm{H}]^{+}$(Calcd for $\left.\mathrm{C}_{10} \mathrm{H}_{21} \mathrm{O}_{4}: 205.1439\right), 187$ $\left[\mathrm{M}-\mathrm{H}_{2} \mathrm{O}+\mathrm{H}\right]^{+}, 169\left[\mathrm{M}-2 \mathrm{H}_{2} \mathrm{O}+\mathrm{H}\right]^{+}$(base). ${ }^{1} \mathrm{H}-\mathrm{NMR}$ (pyridine- $d_{5}, 500$ MHz) $\delta$ : Table $2 .{ }^{13} \mathrm{C}$-NMR (pyridine- $d_{5}, 125 \mathrm{MHz}$ ) $\delta$ : Table 1 .

rel-(1S,2R,4R,8S)-p-Menthane-1,2,8,9-tetrol (7) An amorphous powder, $[\alpha]_{\mathrm{D}}^{23}+11^{\circ}(c=0.1, \mathrm{MeOH})$. Positive FAB-MS $m / z: 243[\mathrm{M}+\mathrm{K}]^{+}, 227$ $[\mathrm{M}+\mathrm{Na}]^{+}, 205.1438[\mathrm{M}+\mathrm{H}]^{+}\left(\right.$Calcd for $\left.\mathrm{C}_{10} \mathrm{H}_{21} \mathrm{O}_{4}: 205.1439\right), 187$ $\left[\mathrm{M}-\mathrm{H}_{2} \mathrm{O}+\mathrm{H}\right]^{+}$(base), $169\left[\mathrm{M}-2 \mathrm{H}_{2} \mathrm{O}+\mathrm{H}\right]^{+}$. ${ }^{1} \mathrm{H}-\mathrm{NMR}$ (pyridine- $d_{5}, 500$ MHz) $\delta$ : Table $2 .{ }^{13} \mathrm{C}$-NMR (pyridine- $d_{5}, 125 \mathrm{MHz}$ ) $\delta$ : Table 1 . HMBC correlations: $\mathrm{H}-2{ }_{\mathrm{ax}} / \mathrm{C}-1, \mathrm{C}-4, \mathrm{C}-6 ; \mathrm{H}-3_{\mathrm{ax}} / \mathrm{C}-4, \mathrm{C}-5, \mathrm{C}-8 ; \mathrm{H}-3$ eq $/ \mathrm{C}-1, \mathrm{C}-5, \mathrm{C}-8 ; \mathrm{H}-$ $4_{\mathrm{ax}} / \mathrm{C}-3, \mathrm{C}-5, \mathrm{C}-6, \mathrm{C}-8, \mathrm{C}-9$; H-5 ${ }_{\mathrm{ax}} / \mathrm{C}-3$, C-4, C-6; H-5 ${ }_{\mathrm{eq}} \mathrm{C}-4, \mathrm{C}-6$; H- ${ }_{\mathrm{ax}} / \mathrm{C}-2$, C-4, C-5, C-7; H-6 ${ }_{\mathrm{eq}} / \mathrm{C}-1, \mathrm{C}-2$, C-4, C-7; $\mathrm{H}_{3}-7 / \mathrm{C}-1, \mathrm{C}-2, \mathrm{C}-6$; $\mathrm{H}_{2}-9 / \mathrm{C}-4, \mathrm{C}-8$, $\mathrm{C}-10 ; \mathrm{H}_{3}-10 / \mathrm{C}-4, \mathrm{C}-8, \mathrm{C}-9$.

rel-(1S,2R,4R,8R)-p-Menthane-1,2,8,9-tetrol (8) An amorphous powder, $[\alpha]_{\mathrm{D}}^{23}+26^{\circ}(c=0.1, \mathrm{MeOH})$. Positive FAB-MS $m / z: 227[\mathrm{M}+\mathrm{Na}]^{+}$, $205.1425[\mathrm{M}+\mathrm{H}]^{+}$(base, Calcd for $\mathrm{C}_{10} \mathrm{H}_{21} \mathrm{O}_{4}: 205.1439$ ), $187[\mathrm{M}-$ $\left.\mathrm{H}_{2} \mathrm{O}+\mathrm{H}\right]^{+}, 169\left[\mathrm{M}-2 \mathrm{H}_{2} \mathrm{O}+\mathrm{H}\right]^{+} .{ }^{1} \mathrm{H}-\mathrm{NMR}$ (pyridine- $\left.d_{5}, 500 \mathrm{MHz}\right) \delta$ : Table $2 .{ }^{13} \mathrm{C}$-NMR (pyridine- $\left.d_{5}, 125 \mathrm{MHz}\right) \delta$ : Table 1 .

rel-(1R,2R,4R,8S)-p-Menthane-1,2,8,9-tetrol (9) An amorphous powder, $[\alpha]_{\mathrm{D}}^{21}-3^{\circ}(c=0.2, \mathrm{MeOH})$. Positive FAB-MS $m / z: 409[2 \mathrm{M}+\mathrm{H}]^{+}, 227$ $[\mathrm{M}+\mathrm{Na}]^{+}, 205.1429[\mathrm{M}+\mathrm{H}]^{+}$(base, Calcd for $\mathrm{C}_{10} \mathrm{H}_{21} \mathrm{O}_{4}: 205.1439$ ), 187 $\left[\mathrm{M}-\mathrm{H}_{2} \mathrm{O}+\mathrm{H}\right]^{+}, 169\left[\mathrm{M}-2 \mathrm{H}_{2} \mathrm{O}+\mathrm{H}\right]^{+} .{ }^{1} \mathrm{H}-\mathrm{NMR}$ (pyridine- $\left.d_{5}, 500 \mathrm{MHz}\right) \delta$ : Table $2 .{ }^{13} \mathrm{C}$-NMR (pyridine- $\left.d_{5}, 125 \mathrm{MHz}\right) \delta$ : Table 1 .

$(1 S, 2 S, 4 R)$ - $p$-Menth-8-ene-1,2-diol 2-O- $\beta$-D-Glucopyranoside (10) Colorless needles $(\mathrm{MeOH}), \mathrm{mp} 154-156^{\circ} \mathrm{C},[\alpha]_{\mathrm{D}}^{25}+13^{\circ}(c=1.7, \mathrm{MeOH})$. Positive FAB-MS $m / z: 665[2 \mathrm{M}+\mathrm{H}]^{+}, 371[\mathrm{M}+\mathrm{K}]^{+}, 355.1731[\mathrm{M}+\mathrm{Na}]^{+}$ (Calcd for $\mathrm{C}_{16} \mathrm{H}_{28} \mathrm{O}_{7} \mathrm{Na}$ : 355.1733 ), $333[\mathrm{M}+\mathrm{H}]^{+}, 315\left[\mathrm{M}-\mathrm{H}_{2} \mathrm{O}+\mathrm{H}\right]^{+}, 153$ $\left[\mathrm{M}-\mathrm{C}_{6} \mathrm{H}_{12} \mathrm{O}_{6}+\mathrm{H}\right]^{+}$(base). ${ }^{1} \mathrm{H}-\mathrm{NMR}$ (pyridine- $\left.d_{5}, 500 \mathrm{MHz}\right) \delta$ : Table 2. ${ }^{13} \mathrm{C}-\mathrm{NMR}$ (pyridine- $\left.d_{5}, 125 \mathrm{MHz}\right) \delta$ : Table 1 . HMBC correlations: $\mathrm{H}-2_{\mathrm{eq}} / \mathrm{C}-$ 1, C-4, C-6, C-7, Glc C-1; H-3 $/$ ax $-1, \mathrm{C}-2, \mathrm{C}-4, \mathrm{C}-5$; H-3 ${ }_{\mathrm{eq}} / \mathrm{C}-1, \mathrm{C}-2, \mathrm{C}-4, \mathrm{C}-$ 5 ; H- 4 ax $/ \mathrm{C}-3, \mathrm{C}-5, \mathrm{C}-6, \mathrm{C}-10$; H-5 $/$ ax $/ \mathrm{C}-1, \mathrm{C}-3, \mathrm{C}-4, \mathrm{C}-6$; H-5 $\mathrm{eq} / \mathrm{C}-1, \mathrm{C}-3, \mathrm{C}-4$, C-6; H- 6 ax $/ \mathrm{C}-5 ; \mathrm{H}-6_{\mathrm{eq}} / \mathrm{C}-1, \mathrm{C}-2$, C-4, C-5, C-7; H 3 -7/C-1, C-2, C-6; H $2-9 / \mathrm{C}-$ 4, C-10; $\mathrm{H}_{3}-10 / \mathrm{C}-4, \mathrm{C}-8$, C-9; Glc H-1/C-2.

Enzymatic Hydrolysis of $\mathbf{1 0}$ A mixture of $\mathbf{1 0}(11 \mathrm{mg})$ and hesperidinase $(5 \mathrm{mg}$, ICN Biomedicals Inc., lot 72635) in water $(5 \mathrm{ml})$ was shaken in a water bath at $37^{\circ} \mathrm{C}$ for $20 \mathrm{~d}$. The mixture was concentrated in vacuo to dryness and the residue was chromatographed over silica gel $\left[\mathrm{CHCl}_{3}-\mathrm{MeOH}-\mathrm{H}_{2} \mathrm{O}(4: 1: 0.1\right.$ and $\left.1: 1: 0.1)\right]$ to afford $1(3 \mathrm{mg})$ and a sugar fraction. The sugar fraction was passed through Sephadex LH-20 (MeOH) to give a syrup, and HPLC [carbohydrate analysis (waters), detector; JASCO RI-930 detector and JASCO OR-990 chiral detector, solv.; $\mathrm{MeCN}-\mathrm{H}_{2} \mathrm{O}$ (17:3), $2 \mathrm{ml} / \mathrm{min} ; t_{\mathrm{R}} 4.50 \mathrm{~min}$ (same location as that of D-glucose)] showed the presence of D-glucose.

$(1 S, 2 S, 4 R, 8 S)$-p-Menthane-1,2,8,9-tetrol $2-O$ - $\beta$-D-Glucopyranoside (11) Colorless needles $(\mathrm{MeOH}), \mathrm{mp} 137-138^{\circ} \mathrm{C},[\alpha]_{\mathrm{D}}^{24}+22^{\circ}(c=1.3$, $\mathrm{MeOH})$. Positive FAB-MS $m / z: 389[\mathrm{M}+\mathrm{Na}]^{+}, 367.1983[\mathrm{M}+\mathrm{H}]^{+}(\mathrm{Calcd}$ for $\left.\mathrm{C}_{16} \mathrm{H}_{31} \mathrm{O}_{9}: 367.1968\right), 349\left[\mathrm{M}-\mathrm{H}_{2} \mathrm{O}+\mathrm{H}\right]^{+}, 187\left[\mathrm{M}-\mathrm{C}_{6} \mathrm{H}_{12} \mathrm{O}_{6}+\mathrm{H}\right]^{+}$ (base). ${ }^{1} \mathrm{H}-\mathrm{NMR}$ (pyridine- $d_{5}, 500 \mathrm{MHz}$ ) $\delta$ : Table $2 .{ }^{13} \mathrm{C}-\mathrm{NMR}$ (pyridine- $d_{5}$, $125 \mathrm{MHz}) \delta$ : Table 1 . HMBC correlations: $\mathrm{H}-2_{\mathrm{eq}} / \mathrm{C}-1, \mathrm{C}-4 ; \mathrm{H}-3_{\mathrm{ax}} / \mathrm{C}-4, \mathrm{C}-5$, $\mathrm{C}-8 ; \mathrm{H}-3_{\mathrm{eq}} / \mathrm{C}-5 ; \mathrm{H}-4_{\mathrm{ax}} / \mathrm{C}-5, \mathrm{C}-9 ; \mathrm{H}-5_{\mathrm{ax}} / \mathrm{C}-6 ; \mathrm{H}^{-} 5_{\mathrm{eq}} / \mathrm{C}-6 ; \mathrm{H}^{-} 6_{\mathrm{ax}} / \mathrm{C}-1, \mathrm{C}-4, \mathrm{C}-5$, C-7; H-6 ${ }_{\mathrm{eq}} / \mathrm{C}-1, \mathrm{C}-2, \mathrm{C}-5 ; \mathrm{H}_{3}-7 / \mathrm{C}-1, \mathrm{C}-2, \mathrm{C}-6 ; \mathrm{H}_{2}-9 / \mathrm{C}-4, \mathrm{C}-8, \mathrm{C}-10 ; \mathrm{H}_{3}-$ 10/C-4, C-8, C-9; Glc H-1/C-2.
Enzymatic Hydrolysis of 11 A mixture of $11(8 \mathrm{mg})$ and $\beta$-glucosidase $(5 \mathrm{mg}$, Toyobo Co., Ltd., lot 52275$)$ in water $(5 \mathrm{ml})$ was shaken in a water bath at $37^{\circ} \mathrm{C}$ for $16 \mathrm{~d}$. The mixture was treated in the same way described for 10 to afford $5(4 \mathrm{mg})$ and a sugar fraction. D-Glucose was detected from the sugar fraction, as described for $\mathbf{1 0}$.

$(1 S, 2 S, 4 R, 8 R)$-p-Menthane-1,2,8,9-tetrol $2-O$ - $\beta$-D-Glucopyranoside (12) An amorphous powder, $[\alpha]_{\mathrm{D}}^{24}+17^{\circ}(c=0.7$, MeOH). Positive FABMS $m / z: 389[\mathrm{M}+\mathrm{Na}]^{+}, \quad 367.1982[\mathrm{M}+\mathrm{H}]^{+}$(Calcd for $\mathrm{C}_{16} \mathrm{H}_{31} \mathrm{O}_{9}$ : 367.1968), $349\left[\mathrm{M}-\mathrm{H}_{2} \mathrm{O}+\mathrm{H}\right]^{+}, 187\left[\mathrm{M}-\mathrm{C}_{6} \mathrm{H}_{12} \mathrm{O}_{6}+\mathrm{H}\right]^{+}$(base). ${ }^{1} \mathrm{H}-\mathrm{NMR}$ (pyridine- $\left.d_{5}, 500 \mathrm{MHz}\right) \delta$ : Table $2 .{ }^{13} \mathrm{C}-\mathrm{NMR}$ (pyridine- $\left.d_{5}, 125 \mathrm{MHz}\right) \delta$ : Table 1. HMBC correlations: $\mathrm{H}-2_{\mathrm{eq}} / \mathrm{C}-1, \mathrm{C}-4, \mathrm{C}-6 ; \mathrm{H}-3_{\mathrm{ax}} / \mathrm{C}-2, \mathrm{C}-4, \mathrm{C}-5, \mathrm{C}-8$; $\mathrm{H}-3_{\mathrm{eq}} / \mathrm{C}-1, \mathrm{C}-2, \mathrm{C}-5, \mathrm{C}-8$; H-4 ${ }_{\mathrm{ax}} / \mathrm{C}-2, \mathrm{C}-3, \mathrm{C}-8, \mathrm{C}-9, \mathrm{C}-10 ; \mathrm{H}-5_{\mathrm{ax}} / \mathrm{C}-1, \mathrm{C}-4$, C-6, C-8; H- ${ }_{\mathrm{eq}} / \mathrm{C}-3$; H- ax $_{1} / \mathrm{C}-1, \mathrm{C}-4, \mathrm{C}-5, \mathrm{C}-7$; H-6 ${ }_{\mathrm{eq}} / \mathrm{C}-1, \mathrm{C}-2, \mathrm{C}-5 ; \mathrm{H}_{3}-7 / \mathrm{C}-$ 1, C-2, C-6; H2 $-9 / \mathrm{C}-4, \mathrm{C}-8, \mathrm{C}-10 ; \mathrm{H}_{3}-10 / \mathrm{C}-4, \mathrm{C}-8$, C-9; Glc H-1/C-2.

Enzymatic Hydrolysis of 12 A mixture of $12(6 \mathrm{mg})$ and $\beta$-glucosidase in water $(5 \mathrm{ml})$ was shaken in a water bath at $37^{\circ} \mathrm{C}$ for $16 \mathrm{~d}$. The mixture was treated in the same way as described for $\mathbf{1 0}$ to afford $\mathbf{6}(3 \mathrm{mg})$ and a sugar fraction. D-Glucose was detected from the sugar fraction, as described for $\mathbf{1 0}$.

rel-(1R,2R,4R,8S)-p-Menthane-1,2,8,9-tetrol 9- $O$ - $\beta$-D-Glucopyranoside (13) An amorphous powder, $[\alpha]_{\mathrm{D}}^{23}-15^{\circ}(c=0.2, \mathrm{MeOH})$. Positive FABMS $m / z: 733[2 \mathrm{M}+\mathrm{H}]^{+}, 405[\mathrm{M}+\mathrm{K}]^{+}, 389.1782[\mathrm{M}+\mathrm{Na}]^{+}$(base, Calcd for $\left.\mathrm{C}_{16} \mathrm{H}_{30} \mathrm{O}_{9} \mathrm{Na}: 389.1788\right), 367[\mathrm{M}+\mathrm{H}]^{+}, 187\left[\mathrm{M}-\mathrm{C}_{6} \mathrm{H}_{12} \mathrm{O}_{6}+\mathrm{H}\right]^{+} .{ }^{1} \mathrm{H}-\mathrm{NMR}$ (pyridine- $\left.d_{5}, 500 \mathrm{MHz}\right) \delta$ : Table $2 .{ }^{13} \mathrm{C}-\mathrm{NMR}$ (pyridine- $\left.d_{5}, 125 \mathrm{MHz}\right) \delta$ : Table 1.

$(1 S, 2 R, 4 R)$ - $p$-Menth-8-ene-2,10-diol 2- $O$ - $\beta$-D-Glucopyranoside (14) An amorphous powder, $[\alpha]_{\mathrm{D}}^{25}-26^{\circ}(c=0.9, \mathrm{MeOH})$. Positive FAB-MS $m / z$ : $665[2 \mathrm{M}+\mathrm{H}]^{+}, 355[\mathrm{M}+\mathrm{Na}]^{+}, 333.1921[\mathrm{M}+\mathrm{H}]^{+}$(base, Calcd for $\mathrm{C}_{16} \mathrm{H}_{29} \mathrm{O}_{7}: 333.1913$ ), $153\left[\mathrm{M}-\mathrm{C}_{6} \mathrm{H}_{12} \mathrm{O}_{6}+\mathrm{H}\right]^{+}$. ${ }^{1} \mathrm{H}-\mathrm{NMR}$ (pyridine- $d_{5}$, $500 \mathrm{MHz}$ ) $\delta$ : Table $2 .{ }^{13} \mathrm{C}-\mathrm{NMR}$ (pyridine- $d_{5}, 125 \mathrm{MHz}$ ) $\delta$ : Table $1 . \mathrm{HMBC}$ correlations: $\mathrm{H}-1_{\mathrm{eq}} / \mathrm{C}-2, \mathrm{C}-3, \mathrm{C}-5, \mathrm{C}-6, \mathrm{C}-7$; $\mathrm{H}-2_{\mathrm{ax}} / \mathrm{C}-1, \mathrm{C}-3, \mathrm{C}-4, \mathrm{C}-6, \mathrm{C}-7$; $\mathrm{H}-3_{\mathrm{ax}} / \mathrm{C}-1, \mathrm{C}-4, \mathrm{C}-5, \mathrm{C}-8 ; \mathrm{H}-3$ eq $/ \mathrm{C}-1, \mathrm{C}-4, \mathrm{C}-5, \mathrm{C}-8 ; \mathrm{H}-4{ }_{\mathrm{ax}} / \mathrm{C}-2, \mathrm{C}-5, \mathrm{C}-6, \mathrm{C}-$ 8, C-9, C-10; H-5 ax /C-1, C-3, C-4, C-8; H-5 ${ }_{\mathrm{eq}} / \mathrm{C}-3, \mathrm{C}-4 ; \mathrm{H}-6_{\mathrm{ax}} / \mathrm{C}-1, \mathrm{C}-4, \mathrm{C}-5$, C-7; H-6 ${ }_{\text {eq }} / \mathrm{C}-1, \mathrm{C}-2$, C-4, C-5, C-7; $\mathrm{H}_{3}-7 / \mathrm{C}-1, \mathrm{C}-6$; H-9a/C-4, C-8, C-10; H9b/ C-4, C-8, C-10; H3-10/C-4, C-8, C-9; Glc H-1/C-2.

Enzymatic Hydrolysis of 14 A mixture of $\mathbf{1 4}(8 \mathrm{mg})$ and $\beta$-glucosidase $(5 \mathrm{mg})$ in water $(5 \mathrm{ml})$ was shaken in a water bath at $37^{\circ} \mathrm{C}$ for $7 \mathrm{~d}$. The mixture was treated in the same way as described for 10 to afford 14a $(4 \mathrm{mg})$ and a sugar fraction. D-Glucose was detected from the sugar fraction, as described for $\mathbf{1 0}$

$(1 S, 2 R, 4 R)-p$-Menth-8-ene-2,10-diol (14a) An amorphous powder, $[\alpha]_{\mathrm{D}}^{22}-11^{\circ}(c=0.3, \mathrm{MeOH})$. Positive FAB-MS $m / z$ : $171[\mathrm{M}+\mathrm{H}]^{+}$(base). ${ }^{1} \mathrm{H}-\mathrm{NMR}$ (pyridine- $\left.d_{5}, 500 \mathrm{MHz}\right) \delta: 5.46(1 \mathrm{H}, \mathrm{d}, J=2.0 \mathrm{~Hz}, \mathrm{H}-9 \mathrm{~b}), 5.09(1 \mathrm{H}$, d, $J=2.0 \mathrm{~Hz}, \mathrm{H}-9 \mathrm{a}), 4.45\left(2 \mathrm{H}\right.$, br s, $\left.\mathrm{H}_{2}-10\right), 4.07(1 \mathrm{H}$, ddd, $J=4.5,4.5$, $\left.12.0 \mathrm{~Hz}, \mathrm{H}-2_{\mathrm{ax}}\right), 2.29\left(1 \mathrm{H}, \mathrm{m}, \mathrm{H}-1_{\mathrm{eq}}\right), 2.28(1 \mathrm{H}, \mathrm{dddd}, J=3.0,3.0,12.0$, $\left.12.0 \mathrm{~Hz}, \mathrm{H}-4_{\mathrm{ax}}\right), 2.13\left(1 \mathrm{H}\right.$, ddd, $\left.J=3.0,4.5,12.0 \mathrm{~Hz}, \mathrm{H}-3_{\mathrm{eq}}\right), 1.86(1 \mathrm{H}, \mathrm{ddd}$, $\left.J=12.0,12.0,12.0 \mathrm{~Hz}, \mathrm{H}-3_{\text {ax }}\right), 1.63(1 \mathrm{H}$, dddd, $J=3.0,3.0,12.0,12.0 \mathrm{~Hz}, \mathrm{H}-$ $\left.6_{\mathrm{ax}}\right), 1.54-1.61\left(2 \mathrm{H}, \mathrm{m}, \mathrm{H}-5_{\mathrm{eq}}, \mathrm{H}-6_{\mathrm{eq}}\right), 1.51(1 \mathrm{H}, \mathrm{dddd}, J=3.0,12.0,12.0$, $12.0 \mathrm{~Hz}, \mathrm{H}-5_{\mathrm{ax}}$ ) $1.19\left(3 \mathrm{H}, \mathrm{d}, J=7.0 \mathrm{~Hz}, \mathrm{H}_{3}-7\right) .{ }^{13} \mathrm{C}-\mathrm{NMR}$ (pyridine- $d_{5}$, $125 \mathrm{MHz}) \delta$ : Table 1.

$(4 R, 6 S)$-7-Hydroxycarveol 7- $O$ - $\beta$-D-Glucopyranoside (15) An amorphous powder, $[\alpha]_{\mathrm{D}}^{24}+12^{\circ}(c=0.4, \mathrm{MeOH})$. Positive FAB-MS $m / z: 661$ $[2 \mathrm{M}+\mathrm{H}]^{+}, 369[\mathrm{M}+\mathrm{K}]^{+}, 331.1755[\mathrm{M}+\mathrm{H}]^{+}$(base, Calcd for $\mathrm{C}_{16} \mathrm{H}_{27} \mathrm{O}_{7}$ : 331.1757), $151\left[\mathrm{M}-\mathrm{C}_{6} \mathrm{H}_{12} \mathrm{O}_{6}+\mathrm{H}\right]^{+} .{ }^{1} \mathrm{H}-\mathrm{NMR}$ (pyridine- $\left.d_{5}, 500 \mathrm{MHz}\right) \delta$ : Table $2 .{ }^{13} \mathrm{C}-\mathrm{NMR}$ (pyridine- $\left.d_{5}, 125 \mathrm{MHz}\right) \delta$ : Table 1 . HNBC correlations: $\mathrm{H}-2{ }_{\mathrm{eq}} / \mathrm{C}-4 ; \mathrm{H}-3{ }_{\mathrm{ax}} / \mathrm{C}-4, \mathrm{C}-5, \mathrm{C}-8 ; \mathrm{H}-3{ }_{\mathrm{eq}} / \mathrm{C}-2, \mathrm{C}-5 ; \mathrm{H}-4_{\mathrm{ax}} / \mathrm{C}-3, \mathrm{C}-5, \mathrm{C}-6, \mathrm{C}-8$, C-9, C-10; H- 5 ax $/ \mathrm{C}-1, \mathrm{C}-4, \mathrm{C}-6, \mathrm{C}-8$; H-5 eq $/ \mathrm{C}-1, \mathrm{C}-3, \mathrm{C}-4, \mathrm{C}-6, \mathrm{C}-8$; H-6/C2, C-4, C-5, C-7; H-7/C-1, C-2, C-6, Glc C-1; H-9a/ C-4, C-8, C-10; H9b/C-4, C-8, C-10; $\mathrm{H}_{3}-10 / \mathrm{C}-4, \mathrm{C}-8$, C-9; Glc H-1/C-7.

Enzymatic Hydrolysis of 15 A mixture of $\mathbf{1 5}(6 \mathrm{mg})$ and $\beta$-glucosidase $(5 \mathrm{mg})$ in water $(5 \mathrm{ml})$ was shaken in a water bath at $37^{\circ} \mathrm{C}$ for $10 \mathrm{~d}$. The mixture was treated in the same way as described for 10 to afford $15 \mathbf{a}(3 \mathrm{mg})$ and a sugar fraction. D-Glucose was detected from the sugar fraction, as described for $\mathbf{1 0}$

$(4 R, 6 S)$-7-Hydroxycarveol (15a) An amorphous powder, $[\alpha]_{D}^{22}+36^{\circ}$ $(c=0.2, \mathrm{MeOH})$. Positive FAB-MS $m / z: 169[\mathrm{M}+\mathrm{H}]^{+}$(base). ${ }^{1} \mathrm{H}-\mathrm{NMR}$ (pyridine- $\left.d_{5}, 500 \mathrm{MHz}\right) \delta: 6.05(1 \mathrm{H}$, br d, $J=4.0 \mathrm{~Hz}, \mathrm{H}-6), 4.80(1 \mathrm{H}, \mathrm{br} \mathrm{s}, \mathrm{H}-$ 9b), $4.77(1 \mathrm{H}, \mathrm{br} \mathrm{s}, \mathrm{H}-9 \mathrm{a}), 4.73\left(1 \mathrm{H}, \mathrm{dd}, J=4.0,4.0 \mathrm{~Hz}, \mathrm{H}-2_{\mathrm{eq}}\right), 4.70(1 \mathrm{H}, \mathrm{d}$, $J=13.0 \mathrm{~Hz}, \mathrm{H}-7 \mathrm{~b}), 4.64(1 \mathrm{H}, \mathrm{d}, J=13.0 \mathrm{~Hz}, \mathrm{H}-7 \mathrm{a}), 2.83(1 \mathrm{H}$, dddd, $J=4.0$, $\left.4.0,13.0,13.0 \mathrm{~Hz}, \mathrm{H}-4_{\mathrm{ax}}\right), 2.21\left(1 \mathrm{H}, \mathrm{ddd}, J=4.0,4.0,13.0 \mathrm{~Hz}, \mathrm{H}-3_{\mathrm{eq}}\right), 2.18$ $\left(1 \mathrm{H}\right.$, ddd, $\left.J=4.0,4.0,17.5 \mathrm{~Hz}, \mathrm{H}-5_{\mathrm{eq}}\right), 1.93(1 \mathrm{H}, \mathrm{brdd}, J=13.0,17.5 \mathrm{~Hz}, \mathrm{H}-$ $\left.5_{\mathrm{ax}}\right), 1.68\left(3 \mathrm{H}, \mathrm{s}, \mathrm{H}_{3}-10\right), 1.65\left(1 \mathrm{H}\right.$, ddd, $\left.J=4.0,13.0,13.0 \mathrm{~Hz}, \mathrm{H}-3_{\mathrm{ax}}\right) \cdot{ }^{13} \mathrm{C}-$ NMR (pyridine- $\left.d_{5}, 125 \mathrm{MHz}\right) \delta$ : Table 1 . 
Acknowledgments The authors thank Mr. Y. Takase and Dr. H. Suzuki of the Analytical Center of Showa Pharmaceutical University for NMR and MS measurements.

\section{References and Notes}

1) Matsumura T., Ishikawa T., Kitajima J., Tetrahedron, 57, 8067-8074 (2001).

2) "Herbal Drugs and Phytopharmaceuticals," ed. by Wichtl M., CRC Press, Stuttgart, 1994, pp. 128-129.

3) Ishikawa T., Sega Y., Kitajima J., Chem. Pharm. Bull., 49, 840-844 (2001).

4) Klyne W., "Determination of Organic Structure by Physical Methods," ed. by Braude E. A., Nachod F. C., Academic Press, New York, 1975, p. 73; Idem, Biochem. J., 47, XIi-XIii (1950).

5) Diaz J. G., Barba B., Herz W., Phytochemistry, 36, 703 -707 (1994); Piatkowski K., Simienuk A., Kuczynski H., Bull. Acad. Pol. Sci. Ser.
Chim., 23, 883-894 (1975).

6) Kitajima J., Aoki Y., Ishikawa T., Tanaka Y., Chem. Pharm. Bull., 47, 639-642 (1999).

7) $\mathrm{CD}$ spectra of $(4 S)-(+)$ - and $(4 R)-(-)$-carvone in $\mathrm{MeOH}$ were as follows: $(+)$-carvone $(c=0.0573 \mathrm{M}) ; \Delta \varepsilon(\mathrm{nm}):-0.41(260),(-)$-carvone $(c=0.0740 \mathrm{M}) ; \Delta \varepsilon(\mathrm{nm}):+0.33(260)$.

8) Kasai R., Suzuo M., Asakawa J., Tanaka O., Tetrahedron Lett., 1977, 175-178; Tori K., Seo S., Yoshimura Y., Arita Y., Tomita Y., ibid., 1977, 179-182; Kasai R., Okihara M., Asakawa J., Mizutani K., Tanaka O., Tetrahedron, 35, 1427-1432 (1979); Mizutani K., Kasai R., Tanaka O., Carbonhydr. Res., 87, 19-26 (1980); Kitajima J., Ishikawa T., Tanaka Y., Chem. Pharm. Bull., 46, 1591-1594 (1998); Ishikawa T., Kitajima J., Tanaka Y., Ono M., Ito Y., Nohara T., ibid., 46, 1738-1742 (1998); Kitajima J., Kimizuka K, Tanaka Y., ibid., 48, $77-80$ (1999).

9) Klein E., Ohloff G., Tetrahedron, 19, 1091-1099 (1963). 